\title{
A track model for railway induced ground vibration resulting from a transition zone
}

\author{
Matthias Germonpré $^{1 *}$, Geert Degrande ${ }^{1}$ and Geert Lombaert ${ }^{1}$
}

\begin{abstract}
To predict ground-borne vibration due to railway traffic, accurate computational models are required. Since these models generally require a substantial computational effort, much research focused on developing computationally efficient methods by either exploiting the regularity of the problem geometry in the direction along the track or assuming a simplified track structure. This paper presents a track modelling approach based on a wave analysis technique for multicoupled periodic structures. The track and part of the soil are modelled with finite elements, while a perfectly matched layer absorbs waves travelling in the semi-infinite soil. A case study is presented investigating a track with transition zone between ballasted and slab track. The track model is verified by comparison with a periodic track model using the Floquet transform. The train-track interaction force and free field vibration are computed for transition zones with gradual and sudden stiffness increase, respectively. It is found that, at higher train speeds, it is more important to have a more gradual stiffness increase in the transition zone.
\end{abstract}

\section{Keywords}

Railway induced vibrations, periodic structures, perfectly matched layer, transition zone

\section{Introduction}

Railway construction in densely populated urban areas has increased the awareness of human discomfort due to railway induced noise and vibration. Accurate numerical models are required to assess noise and vibration problems and to facilitate performance based design of new railway lines ${ }^{1,2}$. Railway induced noise and vibration are generated by a combination of quasistatic and dynamic axle loads. The dynamic component is caused by several excitation mechanisms, such as wheel and track unevenness, impact excitation due to rail joints and wheel flats, and parametric excitation due to stiffness variations in the longitudinal track direction $^{2-4}$.

Several numerical models are currently available to predict ground-borne noise and vibration. The largest flexibility in modelling track and soil is obtained by three-dimensional (3D) coupled finite element-boundary element (FE-BE) models ${ }^{5-7}$. However, these models require extensive modelling effort and computation time. Many authors therefore exploit the regularity of the track geometry to develop simplified track models that are computationally more efficient.

In a so-called 2.5D FE-BE approach, the track is assumed to be invariant in the longitudinal direction. A Fourier transformation of the longitudinal coordinate facilitates an efficient solution in the frequencywavenumber domain. Only a two-dimensional (2D) cross section of the track has to be meshed, allowing for a detailed representation of the cross sectional geometry. This $2.5 \mathrm{D}$ approach is used to study vibrations induced by wheel and track unevenness and has been developed for tracks at grade $^{8-10}$ as well as in tunnels ${ }^{9-12}$. However, these models cannot take into account parametric excitation as arising, for example, from the periodic rail support in ballasted tracks. Furthermore, the loads transferred by the sleepers to the ballast are distributed along the track resulting in an incorrect stress distribution under the sleepers.

Alternatively, the track can be assumed to be periodic and a Floquet transformation ${ }^{13,14}$ enables a solution based on the discretization of a single 3D periodic cell. This approach allows to account for parametric excitation due to the periodic rail support by sleepers, but cannot be used to model other sources of track stiffness variations, such as hanging sleepers, transition zones or spatial variations of the subgrade stiffness. Arlaud et al. ${ }^{15,16}$ developed a similar model, where the track is divided into periodic cells of which the modes are computed, thus significantly reducing the number of degrees of freedom compared to a 3D finite element model. This model can be used to account for other sources of track stiffness variations as well, but this reduces the computational efficiency. It is very efficient to predict the response of the track or receivers close to the track, but less suited for the prediction of the response at larger distances from the track, since the soil domain is modelled with fixed boundaries and therefore no absorption of waves is accounted for at these boundaries.

For the study of dynamic train-track interaction, more simplified track models have been developed ${ }^{17}$ where the soil is modelled by a series of masses, springs and dampers. These models do not allow to compute the vibration transfer to the free field. Therefore, they are often used in two-step approaches in which the train-track interaction problem is solved first and the vibration transfer is considered in the second step ${ }^{18,19}$. An important disadvantage is the need for two different

${ }^{1} \mathrm{KU}$ Leuven, Department of Civil Engineering, Belgium

Matthias Germonpré, KU Leuven, Department of Civil Engineering, Kasteelpark Arenberg 40, 3001 Leuven, Belgium. 
track models, which can introduce modelling errors. For the study of transition zones, simplified train-track interaction models calibrated with field measurements are also used ${ }^{20,21}$.

This paper presents a general two-step train-tracksoil coupling algorithm. The advantage of the algorithm is that no assumption is made on the track geometry; it can either be invariant or completely irregular in the longitudinal direction. Also, a novel track modelling approach based on a wave analysis technique for multicoupled periodic structures ${ }^{22}$ is presented. The track and part of the soil are modelled with finite elements and wave reflection on the boundaries of the soil domain is avoided by means of a perfectly matched layer ${ }^{23}$. The advantage of this technique is that it allows to model any kind of track stiffness variation while it is computationally much more efficient than a full 3D model. The first section describes the two-step coupling algorithm and the track model based on wave analysis for multi-coupled periodic structures. Next, this track model is used in a case study of a track with transition zone between ballasted and slab track. The proposed track modelling approach is verified by comparing the vertical rail receptance and track-free field mobility for this model and a periodic model based on the Floquet transform. The train-track interaction force and free field vibration are computed for transition zones with gradual and sudden stiffness increase, respectively.

\section{Methodology}

\section{Train-track-soil coupling algorithm}

In this subsection, a general two-step algorithm to solve the train-track-soil interaction problem is presented. First, the train-track interaction problem is solved in the time domain to predict the axle loads. Next, these axle loads are used to predict the free field vibrations in the frequency domain.

In the first step of the algorithm, the train-track interaction problem is solved by an iterative sequential Dirichlet-Neumann algorithm ${ }^{24}$ in the time domain (figure 1). Continuity of displacements and equilibrium of forces are enforced at the wheel-rail contact points. The equations are solved for the train and track separately until convergence is reached.

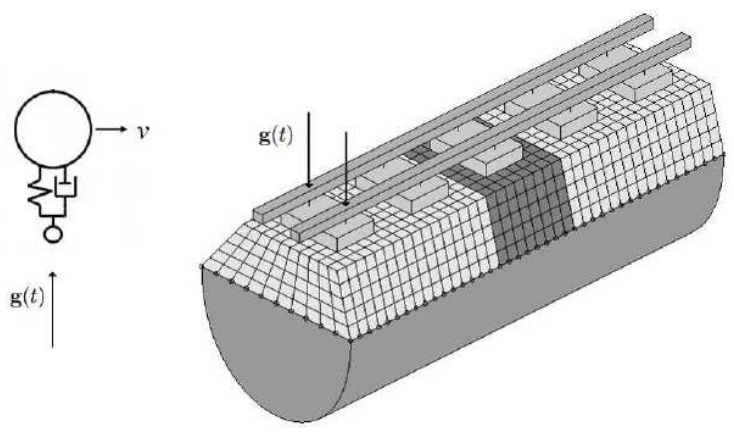

Figure 1. Step 1: train-track coupling in the time domain.
The train system of equations is:

$$
\begin{array}{r}
{\left[\mathbf{K}_{\mathrm{v}}+\frac{\delta}{\alpha \Delta t} \mathbf{C}_{\mathrm{v}}+\frac{1}{\alpha \Delta t^{2}} \mathbf{M}_{\mathrm{v}}\right] \mathbf{u}_{\mathrm{v}}(t)=\mathbf{F}_{\mathrm{v}}(t)+} \\
{\left[\left(\frac{1}{2 \alpha}-1\right) \mathbf{M}_{\mathrm{v}}+\frac{\Delta t}{2}\left(\frac{\delta}{\alpha}-1\right) \mathbf{C}_{\mathrm{v}}\right] \ddot{\mathbf{u}}_{\mathrm{v}}(t-\Delta t)+} \\
{\left[\frac{1}{\alpha \Delta t} \mathbf{M}_{\mathrm{v}}+\left(\frac{\delta}{\alpha}-1\right) \mathbf{C}_{\mathrm{v}}\right] \dot{\mathbf{u}}_{\mathrm{v}}(t-\Delta t)+} \\
{\left[\frac{1}{\alpha \Delta t^{2}} \mathbf{M}_{\mathrm{v}}+\frac{\delta}{\alpha \Delta t} \mathbf{C}_{\mathrm{v}}\right] \mathbf{u}_{\mathrm{v}}(t-\Delta t)}
\end{array}
$$

with Newmark parameters $\alpha$ and $\delta$ and time step $\Delta t$. The matrices $\mathbf{K}_{\mathrm{v}}, \mathbf{C}_{\mathrm{v}}$ and $\mathbf{M}_{\mathrm{v}}$ are the stiffness, damping and mass matrices of the train, respectively. The vector $\mathbf{u}_{\mathrm{v}}(t)=\left\{\mathbf{u}_{\mathrm{b}}(t) ; \mathbf{u}_{\mathrm{a}}(t)\right\}$ contains the degrees of freedom of the train, with $\mathbf{u}_{\mathrm{a}}(t)$ the vertical displacements of the wheel-rail contact points and $\mathbf{u}_{\mathrm{b}}(t)$ the remaining degrees of freedom. The vector $\mathbf{F}_{\mathrm{v}}(t)=$ $\{\mathbf{0} ; \mathbf{g}(t)\}$ contains the axle loads $\mathbf{g}(t)$ between the wheels and rails. A Dirichlet boundary condition is used for the train, imposing the axle displacements $\mathbf{u}_{\mathrm{a}}(t)$ to compute the axle loads $\mathbf{g}(t)$.

For the track, a Neumann boundary condition is used. The vertical track displacements $u_{\mathrm{t}}(\mathbf{x}, t)$ are calculated as the convolution of the time history of the $n_{\mathrm{a}}$ axle loads $g_{k}(t)$ and transfer functions $h_{z z}\left(\mathbf{x}_{k}(\tau), \mathbf{x}^{\prime}, t-\tau\right)$ relating the displacement at $\mathbf{x}^{\prime}$ in the direction $\mathbf{e}_{z}$ at time $t-\tau$ due to a unit load applied at $\mathbf{x}_{k}(\tau)$ in the direction $\mathbf{e}_{z}$ at time 0 :

$$
u_{\mathrm{t}}\left(\mathbf{x}^{\prime}, t\right)=\sum_{k=1}^{n_{a}} \int_{0}^{t} h_{z z}\left(\mathbf{x}_{k}(\tau), \mathbf{x}^{\prime}, t-\tau\right) g_{k}(\tau) \mathrm{d} \tau .
$$

Only vertical interaction forces between the train and the track are considered in equation (2). The transfer functions $h_{z z}\left(\mathbf{x}_{k}(\tau), \mathbf{x}^{\prime}, t-\tau\right)$ are computed by means of a track model. The time-dependent positions of the axles are denoted by $\mathbf{x}_{k}(\tau)=\left\{x_{k 0}, y_{k 0}+v \tau, z_{k 0}\right\}^{\mathrm{T}}$, with $\left\{x_{k 0}, y_{k 0}, z_{k 0}\right\}^{\mathrm{T}}$ the initial position of axle $k$ and $v$ the train speed.

The train axles are in perfect contact with the track. Accounting for the combined wheel and rail unevenness $u_{\mathrm{w} / \mathrm{r}}(t)$, the following expression holds for each axle $k^{25}$ :

$$
u_{\mathrm{a} k}(t)=u_{\mathrm{t} k}(t)+u_{\mathrm{w} / \mathrm{r} k}(t),
$$

where $u_{\mathrm{t} k}(t)$ is the vertical displacement of the track at the position of the $k$-th axle and $u_{\mathrm{w} / \mathrm{r} k}(t)$ is the unevenness perceived by this axle.

The train-track interaction problem is solved by means of an iterative sequential Dirichlet-Neumann algorithm that proceeds as follows for every time $t=$ $n_{\mathrm{t}} \Delta t$. First it is assumed that $\ddot{u}_{\mathrm{a} k}(t)=\ddot{u}_{\mathrm{a} k}(t-\Delta t)$ for each axle $k$ and the axle displacements $u_{\mathrm{a} k}(t)$ are calculated with Newmark's method:

$$
\begin{aligned}
u_{\mathrm{a} k}(t)= & u_{\mathrm{a} k}(t-\Delta t)+\dot{u}_{\mathrm{a} k}(t-\Delta t) \Delta t+ \\
& \left(\frac{1}{2}-\alpha\right) \ddot{u}_{\mathrm{a} k}(t-\Delta t) \Delta t^{2}+\alpha \ddot{u}_{\mathrm{a} k}(t) \Delta t^{2} \\
= & u_{\mathrm{a} k}(t-\Delta t)+\dot{u}_{\mathrm{a} k}(t-\Delta t) \Delta t+ \\
& \frac{1}{2} \ddot{u}_{\mathrm{a} k}(t) \Delta t^{2}
\end{aligned}
$$


Next, the following steps are repeated for every iteration $n$ until convergence is achieved for every axle $k$ at this time step $t$ :

1. Knowing the axle displacements $u_{\mathrm{a} k}^{n}(t)$, equation (1) is used to compute the displacements $\mathbf{u}_{\mathrm{b}}^{n}(t)$ and the axle loads $\mathbf{g}^{n}(t)$.

2. Compute the vertical track displacement $u_{\mathrm{t}}^{n}\left(\mathbf{x}^{\prime}, t\right)$ from equation (2) in the discrete time domain:

$$
\begin{array}{r}
u_{\mathrm{t}}^{n}\left(\mathbf{x}^{\prime}, t\right)=u_{\mathrm{t}}^{n}\left(\mathbf{x}^{\prime}, n_{\mathrm{t}} \Delta t\right) \simeq \\
\sum_{k=1}^{n_{\mathrm{a}}} \sum_{j=0}^{n_{\mathrm{t}}} h_{z z}\left(\mathbf{x}_{k}(j \Delta t), \mathbf{x}^{\prime},\left(n_{\mathrm{t}}-j\right) \Delta t\right) g_{k}(j \Delta t) \Delta t .
\end{array}
$$

All axle loads $g_{k}(j \Delta t)$ for $j<n_{\mathrm{t}}$ are known, while $g_{k}\left(n_{\mathrm{t}} \Delta t\right)$ are the axle loads $\mathbf{g}^{n}(t)$ obtained in step 1. Evaluating equation (5) yields the track displacements $u_{\mathrm{t} k}^{n}(t)$ at each axle $k$.

3. From $u_{\mathrm{t} k}^{n}(t)$, the axle displacements $u_{\mathrm{a} k \text {, unrelaxed }}^{n+1}(t)$ are computed with equation (3). A relaxation technique $^{26}$ is used to calculate relaxed values for $\mathbf{u}_{\mathrm{a}}^{n+1}(t)$ :

$$
\begin{aligned}
\mathbf{u}_{\mathrm{a}}^{n+1}(t) & =\lambda^{n}(t) \mathbf{u}_{\mathrm{a}, \text { unrelaxed }}^{n+1}(t) \\
& +\left(1-\lambda^{n}(t)\right) \mathbf{u}_{\mathrm{a}}^{n}(t) .
\end{aligned}
$$

The relaxation parameter $\lambda^{n}(t)$ is defined as:

$$
\lambda^{n}(t)=-\lambda^{n-1}(t) \frac{\left(\mathbf{r}^{n}(t)-\mathbf{r}^{n-1}(t)\right)^{\mathrm{T}} \mathbf{r}^{n-1}(t)}{\| \mathbf{r}^{n}(t)-\mathbf{r}^{n-1}(t)||^{2}},
$$

with the residual $\mathbf{r}^{n}(t)$ defined as:

$$
\mathbf{r}^{n}(t)=\mathbf{u}_{\mathrm{a}}^{n}(t)-\mathbf{u}_{\mathrm{a}, \text { unrelaxed }}^{n+1}(t) .
$$

The iteration procedure is terminated if the following convergence criterion with accuracy $\epsilon$ holds for every axle $k$ :

$$
\left|\frac{u_{\mathrm{a} k}^{n+1}(t)-u_{\mathrm{a} k}^{n}(t)}{u_{\mathrm{a} k}^{n+1}(t)}\right|<\epsilon .
$$

An accuracy of $10^{-10}$ is used. The algorithm converges on average after 3 or 4 iterations for a train axle or train car model, respectively.

In the second step of the train-track-soil coupling algorithm, the axle loads $\mathbf{g}(t)$ are used to compute the displacements at the track-soil interface. Next, the tractions at this interface are computed and a boundary element model of the soil is used to obtain the vibrations in the free field (figure 2). The axle load distribution on the track is collected in the body force $\rho b_{z}(\mathbf{x}, t)$ as $^{27}$ :

$\rho b_{z}(\mathbf{x}, t)=\sum_{k=1}^{n_{a}} \delta\left(x-x_{k 0}\right) \delta\left(y-y_{k 0}-v t\right) \delta\left(z-z_{k 0}\right) g_{k}(t)$

The body force $\rho b_{z}(\mathbf{x}, t)$ is transformed to the frequency domain and applied on the track domain $\Omega_{\mathrm{t}}$ to predict the displacements $\hat{u}_{\mathrm{ts} i}\left(\mathbf{x}^{\prime}, \omega\right)$ at the track-soil

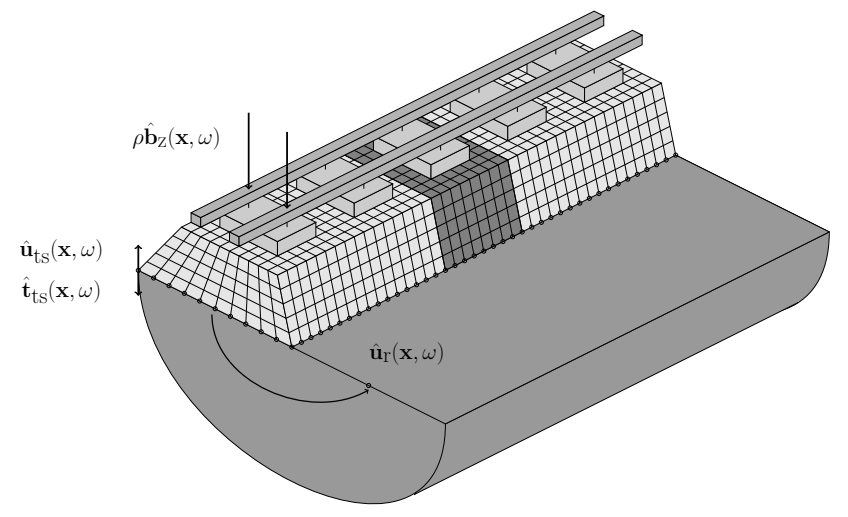

Figure 2. Step 2: propagation of waves from track to free field.

interface:

$$
\hat{u}_{\mathrm{ts} i}\left(\mathbf{x}^{\prime}, \omega\right)=\int_{\Omega_{\mathrm{t}}} \hat{h}_{z i}\left(\mathbf{x}, \mathbf{x}^{\prime}, \omega\right) \rho \hat{b}_{z}(\mathbf{x}, \omega) \mathrm{d} \Omega_{\mathrm{t}}
$$

where $\hat{h}_{z i}\left(\mathbf{x}, \mathbf{x}^{\prime}, \omega\right)$ are the transfer functions that relate the displacement at point $\mathbf{x}^{\prime}$ on the track-soil interface in the direction $\mathbf{e}_{i}$ to a load in point $\mathbf{x}$ on the track in the direction $\mathbf{e}_{z}{ }^{1}$.

If the soil is modelled as a horizontally layered halfspace and is therefore invariant in the longitudinal track direction, the free field vibration can be computed with a $2.5 \mathrm{D}$ boundary element model of the soil based on the dynamic representation theorem of elastodynamics ${ }^{28}$. As a result, the periodic model with PML is not needed in this step and the free field vibrations can be computed at much larger distances from the track than the width of the FE model used in step 1. The displacements at the track-soil interface are transformed to the wavenumber domain by means of a Fourier transform:

$$
\underline{\tilde{\mathbf{u}}}_{\mathrm{ts}}\left(k_{y}, \omega\right)=\int_{-\infty}^{\infty} \underline{\hat{\mathbf{u}}}_{\mathrm{ts}}(y, \omega) e^{+\mathrm{i} k_{y} y} \mathrm{~d} y .
$$

The displacements $\underline{\underline{\mathbf{u}}}_{\mathrm{ts}}\left(k_{y}, \omega\right)$ are used to compute the tractions $\underline{\tilde{\mathbf{t}}}_{\mathrm{ts}}\left(k_{y}, \omega\right)$ at the track-soil interface using the boundary element equations ${ }^{9}$ :

$$
\tilde{\mathbf{t}}_{\mathrm{ts}}\left(k_{y}, \omega\right)=\tilde{\mathbf{U}}^{-1}\left(k_{y}, \omega\right)\left(\tilde{\mathbf{T}}\left(k_{y}, \omega\right)+\mathbf{I}\right) \underline{\mathbf{u}}_{\mathrm{ts}}\left(k_{y}, \omega\right),
$$

with $\tilde{\mathbf{U}}\left(k_{y}, \omega\right)$ and $\tilde{\mathbf{T}}\left(k_{y}, \omega\right)$ the boundary element system matrices. The free field vibrations $\underline{\underline{\mathbf{u}}}_{\mathrm{r}}\left(k_{y}, \omega\right)$ are computed:

$$
\underline{\tilde{\mathbf{u}}}_{\mathrm{r}}\left(k_{y}, \omega\right)=\tilde{\mathbf{U}}_{\mathrm{r}}\left(k_{y}, \omega\right) \tilde{\mathbf{t}}_{\mathrm{ts}}\left(k_{y}, \omega\right)-\tilde{\mathbf{T}}_{\mathrm{r}}\left(k_{y}, \omega\right) \underline{\tilde{\mathbf{u}}}_{\mathrm{ts}}\left(k_{y}, \omega\right),
$$

with $\tilde{\mathbf{U}}_{\mathrm{r}}\left(k_{y}, \omega\right)$ and $\tilde{\mathbf{T}}_{\mathrm{r}}\left(k_{y}, \omega\right)$ the boundary element transfer matrices. The free field vibrations in the spatial domain are calculated by an inverse Fourier transform:

$$
\underline{\tilde{\mathbf{u}}}_{\mathrm{r}}(y, \omega)=\frac{1}{2 \pi} \int_{-\infty}^{\infty} \underline{\tilde{\mathbf{u}}}_{\mathrm{r}}\left(k_{y}, \omega\right) e^{-\mathrm{i} k_{y} y} \mathrm{~d} k_{y} .
$$


The advantage of the coupling algorithm is that no assumption is made on the track geometry. Any track geometry can be modelled, as long as a track model is available to compute the transfer functions $h_{z z}\left(\mathbf{x}, \mathbf{x}^{\prime}, t\right)$ and $\hat{h}_{z i}\left(\mathbf{x}, \mathbf{x}^{\prime}, \omega\right)$ in equations (5) and (11). In the following subsection, the computation of these transfer functions is discussed for a track with varying characteristics in the longitudinal direction, e.g. a track with transition zone.

\section{Track model based on wave analysis of multi-coupled periodic structures}

In this subsection, the computation of the transfer functions $h_{z z}\left(\mathbf{x}, \mathbf{x}^{\prime}, t\right)$ and $\hat{h}_{z i}\left(\mathbf{x}, \mathbf{x}^{\prime}, \omega\right)$ is discussed. These transfer functions can be computed by means of a 3D finite element-boundary element (FE-BE) model, modelling the superstructure by finite elements and the semi-infinite soil domain by boundary elements. In order to reduce the computation time, the track geometry can be assumed to be periodic in the longitudinal direction $\mathbf{e}_{y}$ and a Floquet transform can be used ${ }^{13,14}$. This approach is computationally very efficient, but does not allow to consider a track with varying stiffness in the longitudinal direction such as tracks with transition zones, hanging sleepers or spatial variations of the ballast and subgrade stiffness. Therefore, an alternative approach is presented, based on the wave analysis technique for multi-coupled periodic structures developed by Mead ${ }^{22}$, where the track is modeled as a series of cells. The advantage of this approach is that it can take into account track geometry changes in the longitudinal direction, unlike a periodic or $2.5 \mathrm{D}$ model, while the number of degrees of freedom never exceeds the number of degrees of freedom of one cell. Therefore, this approach significantly reduces the memory requirements compared to a $3 \mathrm{D}$ FE-BE model.

Consider a track divided into $M$ parts with different properties (figure 3), with a central region of $M-2$ parts and semi-infinite series of cells extending infinitely to the left (part 1) and to the right (part $M$ ). Each part $j$ of the track consists of $N_{j}$ periodic cells with length $L_{j}$. The parts are separated by $M-1$ interfaces.

When the track is excited by a force $\mathbf{F}$ applied on a cell of part $j$, the forced vibration in the entire track structure can be studied by investigating the free wave propagation in all cells left and right from the excited cell.

The dynamic stiffness matrix of one cell is calculated as:

$$
\mathbf{S}=\mathbf{K}+i \omega \mathbf{C}-\omega^{2} \mathbf{M}
$$

with $\mathbf{K}, \mathbf{C}$ and $\mathbf{M}$ the stiffness, damping and mass matrix of the cell. The displacement and force vectors $\mathbf{u}$ and $\mathbf{F}$ of each cell are partitioned into three parts: the nodes on the left boundary, the interior nodes and the nodes on the right boundary, referred to by the indices L, I and R, respectively. These vectors are related through the dynamic stiffness matrix $\mathbf{S}$ of the cell:

$$
\left[\begin{array}{ccc}
\mathbf{S}_{\mathrm{LL}} & \mathbf{S}_{\mathrm{LI}} & \mathbf{S}_{\mathrm{LR}} \\
\mathbf{S}_{\mathrm{IL}} & \mathbf{S}_{\mathrm{II}} & \mathbf{S}_{\mathrm{IR}} \\
\mathbf{S}_{\mathrm{RL}} & \mathbf{S}_{\mathrm{RI}} & \mathbf{S}_{\mathrm{RR}}
\end{array}\right]\left\{\begin{array}{c}
\mathbf{u}_{\mathrm{L}} \\
\mathbf{u}_{\mathrm{I}} \\
\mathbf{u}_{\mathrm{R}}
\end{array}\right\}=\left\{\begin{array}{c}
\mathbf{F}_{\mathrm{L}} \\
\mathbf{F}_{\mathrm{I}} \\
\mathbf{F}_{\mathrm{R}}
\end{array}\right\}
$$

Consider an infinite series of this cell with a free wave traveling through cell $k$ on which no external force is applied (figure 4). After condensation of the interior degrees of freedom, the following equation holds:

$$
\left[\begin{array}{ll}
\tilde{\mathbf{S}}_{\mathrm{LL}} & \tilde{\mathbf{S}}_{\mathrm{LR}} \\
\tilde{\mathbf{S}}_{\mathrm{RL}} & \tilde{\mathbf{S}}_{\mathrm{RR}}
\end{array}\right]\left\{\begin{array}{l}
\mathbf{u}_{\mathrm{L} k} \\
\mathbf{u}_{\mathrm{R} k}
\end{array}\right\}=\left\{\begin{array}{l}
\mathbf{F}_{\mathrm{L} k} \\
\mathbf{F}_{\mathrm{R} k}
\end{array}\right\}
$$

where a tilde indicates a matrix after condensation of the interior degrees of freedom.

The displacements and forces at the left-hand side of cells $k$ and $k+1$ are related by the propagation constant $\mu$ of the free wave:

$$
\begin{gathered}
\mathbf{u}_{\mathrm{L} k+1}=e^{-\mu} \mathbf{u}_{\mathrm{L} k}, \\
\mathbf{F}_{\mathrm{L} k+1}=e^{-\mu} \mathbf{F}_{\mathrm{L} k} .
\end{gathered}
$$

At the interface between cells $k$ and $k+1$, continuity of displacements is enforced. Combining this with equation (19) yields a relationship between the displacements at the left- and right-hand side of cell $k$ :

$$
\mathbf{u}_{\mathrm{R} k}=\mathbf{u}_{\mathrm{L} k+1}=e^{-\mu} \mathbf{u}_{\mathrm{L} k}
$$

Similarly, a relationship between the forces at the leftand right-hand side of cell $k$ is obtained after enforcing equilibrium of forces at the interface between cells $k$ and $k+1$ :

$$
\mathbf{F}_{\mathrm{R} k}=-\mathbf{F}_{\mathrm{L} k+1}=-e^{-\mu} \mathbf{F}_{\mathrm{L} k} .
$$

Inserting equations (18) into equation (22) yields:

$$
\tilde{\mathbf{S}}_{\mathrm{RL}} \mathbf{u}_{\mathrm{L} k}+\tilde{\mathbf{S}}_{\mathrm{RR}} \mathbf{u}_{\mathrm{R} k}=-e^{-\mu}\left(\tilde{\mathbf{S}}_{\mathrm{LL}} \mathbf{u}_{\mathrm{L} k}+\tilde{\mathbf{S}}_{\mathrm{LR}} \mathbf{u}_{\mathrm{R} k}\right) .
$$

For each cell type, the free wave eigenvectors and propagation constants are found from the following generalized eigenvalue problem, obtained by combining equations (21) and (23):

$$
\left[\begin{array}{cc}
\tilde{\mathbf{S}}_{\mathrm{RL}} & \tilde{\mathbf{S}}_{\mathrm{RR}} \\
\mathbf{0} & \mathbf{I}
\end{array}\right]\left\{\begin{array}{l}
\mathbf{u}_{\mathrm{L} k} \\
\mathbf{u}_{\mathrm{R} k}
\end{array}\right\}=e^{-\mu}\left[\begin{array}{cc}
-\tilde{\mathbf{S}}_{\mathrm{LL}} & -\tilde{\mathbf{S}}_{\mathrm{LR}} \\
\mathbf{I} & \mathbf{0}
\end{array}\right]\left\{\begin{array}{l}
\mathbf{u}_{\mathrm{L} k} \\
\mathbf{u}_{\mathrm{R} k}
\end{array}\right\} .
$$

Both sides of equation (24) are multiplied by the inverse of the matrix at the right-hand side of this equation. Using the properties of a block matrix, this results in:

$$
\left[\begin{array}{cc}
\mathbf{0} & \mathbf{I} \\
-\tilde{\mathbf{S}}_{\mathrm{LR}}^{-1} \tilde{\mathbf{S}}_{\mathrm{RL}} & -\tilde{\mathbf{S}}_{\mathrm{LR}}^{-1}\left(\tilde{\mathbf{S}}_{\mathrm{LL}}+\tilde{\mathbf{S}}_{\mathrm{RR}}\right)
\end{array}\right]\left\{\begin{array}{l}
\mathbf{u}_{\mathrm{L} k} \\
\mathbf{u}_{\mathrm{R} k}
\end{array}\right\}=e^{-\mu}\left\{\begin{array}{c}
\mathbf{u}_{\mathrm{L} k} \\
\mathbf{u}_{\mathrm{R} k}
\end{array}\right\} .
$$

If needed, the condition of the coefficient matrix in this equation can be improved by multiplying the unit matrix with an appropriate integer number. Alternatively, the following polynomial eigenvalue problem can be solved to obtain the free wave eigenvectors and propagation constants:

$$
\left(\tilde{\mathbf{S}}_{\mathrm{RL}}+e^{-\mu}\left(\tilde{\mathbf{S}}_{\mathrm{LL}}+\tilde{\mathbf{S}}_{\mathrm{RR}}\right)+e^{-2 \mu} \tilde{\mathbf{S}}_{\mathrm{LR}}\right) \mathbf{u}_{\mathrm{L} k}=0 .
$$

At the boundary between any two cells, $n_{\mathrm{c}}$ degrees of freedom exist. Therefore, $n_{\mathrm{c}}$ free waves can propagate in both directions, each wave characterized by its wave eigenvector and propagation constant $\mu$. The complex propagation constants are found from the 


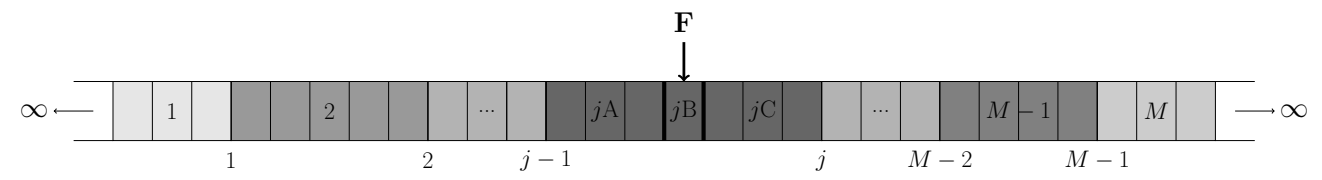

Figure 3. Track consisting of $M$ types of track structure, separated by $M-1$ interfaces, and partition of part $j$.

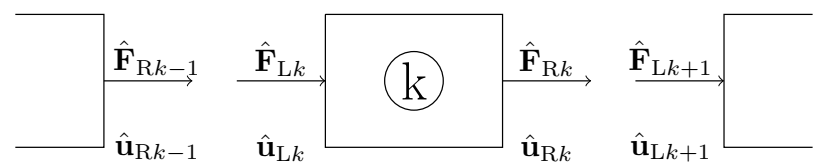

Figure 4. Displacements and forces on cell $k$.

eigenvalues $e^{-\mu}$ of equation (25). Propagation constants with positive real part belong to the positive-going waves, while those with negative real part belong to the negative-going waves. Each propagation constant $\mu$ is associated with a normalized eigenvector $\boldsymbol{\psi}$ and a normalized force vector $\boldsymbol{\rho}$. The force vectors are calculated by inserting equation (21) into equation (18)

$$
\boldsymbol{\rho}=\left[\tilde{\mathbf{S}}_{\mathrm{LL}}+\tilde{\mathbf{S}}_{\mathrm{LR}} e^{-\mu}\right] \boldsymbol{\psi}
$$

for positive-going waves, and

$$
\boldsymbol{\rho}=\left[\tilde{\mathbf{S}}_{\mathrm{RR}}+\tilde{\mathbf{S}}_{\mathrm{RL}} e^{\mu}\right] \boldsymbol{\psi}
$$

for negative-going waves. For each cell type $j$, the vectors $\boldsymbol{\psi}$ and $\boldsymbol{\rho}$ are collected in the matrices $\boldsymbol{\Psi}_{\mathrm{p}}^{j}$ and $\mathbf{R}_{\mathrm{p}}^{j}$ for positive-going waves and $\boldsymbol{\Psi}_{\mathrm{n}}^{j}$ and $\mathbf{R}_{\mathrm{n}}^{j}$ for negativegoing waves. In free vibration, the displacements and forces at the interface between two cells of type $j$ are always a linear combination of the eigenmodes $\boldsymbol{\Psi}_{\mathrm{p}}^{j}$ and $\boldsymbol{\Psi}_{\mathrm{n}}^{j}$ and forces $\mathbf{R}_{\mathrm{p}}^{j}$ and $\mathbf{R}_{\mathrm{n}}^{j}$, respectively.

In the track structure shown in figure 3 , part 1 consists of a semi-infinite series of cells extending infinitely to the left. All the waves in this part are negative-going. At the right-hand side of part 1 , the displacements $\mathbf{u}_{\mathrm{R}}^{1}$ and forces $\mathbf{F}_{\mathrm{R}}^{1}$ are decomposed into linear combinations of the eigenmodes $\boldsymbol{\Psi}_{\mathrm{n}}^{1}$ and forces $\mathbf{R}_{\mathrm{n}}^{1}$, respectively:

$$
\begin{gathered}
\mathbf{u}_{\mathrm{R}}^{1}=\boldsymbol{\Psi}_{\mathrm{n}}^{1} \boldsymbol{\alpha}_{\mathrm{n}}^{1}, \\
\mathbf{F}_{\mathrm{R}}^{1}=\mathbf{R}_{\mathrm{n}}^{1} \boldsymbol{\alpha}_{\mathrm{n}}^{1},
\end{gathered}
$$

with $\boldsymbol{\alpha}_{\mathrm{n}}^{1}$ the modal coordinates of the negative-going waves in part 1 . Eliminating $\boldsymbol{\alpha}_{\mathrm{n}}^{1}$ from equations (29) and (30) results in the following expression:

$$
\mathbf{F}_{\mathrm{R}}^{1}=\mathbf{R}_{\mathrm{n}}^{1}\left(\boldsymbol{\Psi}_{\mathrm{n}}^{1}\right)^{-1} \mathbf{u}_{\mathrm{R}}^{1}=\mathbf{S}_{\mathrm{R}}^{1} \mathbf{u}_{\mathrm{R}}^{1}
$$

with $\mathbf{S}_{\mathrm{R}}^{1}$ the dynamic stiffness matrix of the semi-infinite part 1 . This procedure can be repeated for the semiinfinite part $M$ of the track:

$$
\mathbf{F}_{\mathrm{L}}^{\mathrm{M}}=\mathbf{R}_{\mathrm{p}}^{\mathrm{M}}\left(\boldsymbol{\Psi}_{\mathrm{p}}^{\mathrm{M}}\right)^{-1} \mathbf{u}_{\mathrm{L}}^{\mathrm{M}}=\mathbf{S}_{\mathrm{L}}^{\mathrm{M}} \mathbf{u}_{\mathrm{L}}^{\mathrm{M}}
$$

with $\mathbf{S}_{\mathrm{L}}^{\mathrm{M}}$ the dynamic stiffness matrix of part $M$.

In parts $j$ from 2 to $M-1$, both positiveand negative-going waves propagate. The forces and displacements at the left- and right-hand side of part $j$ can be written as:

$$
\begin{gathered}
\left\{\begin{array}{c}
\mathbf{u}_{\mathrm{L}}^{j} \\
\mathbf{u}_{\mathrm{R}}^{j}
\end{array}\right\}=\left[\begin{array}{cc}
\boldsymbol{\Psi}_{\mathrm{p}}^{j} & \boldsymbol{\Psi}_{\mathrm{n}}^{j} e^{N_{j} \mu_{\mathrm{n}}^{j}} \\
\boldsymbol{\Psi}_{\mathrm{p}}^{j} e^{-N_{j} \mu_{\mathrm{p}}^{j}} & \boldsymbol{\Psi}_{\mathrm{n}}^{j}
\end{array}\right]\left\{\begin{array}{l}
\boldsymbol{\alpha}_{\mathrm{p}}^{j} \\
\boldsymbol{\alpha}_{\mathrm{n}}^{j}
\end{array}\right\}, \\
\left\{\begin{array}{l}
\mathbf{F}_{\mathrm{L}}^{j} \\
\mathbf{F}_{\mathrm{R}}^{j}
\end{array}\right\}=\left[\begin{array}{cc}
\mathbf{R}_{\mathrm{p}}^{j} & -\mathbf{R}_{\mathrm{n}}^{j} e^{N_{j} \mu_{\mathrm{n}}^{j}} \\
-\mathbf{R}_{\mathrm{p}}^{j} e^{-N_{j} \mu_{\mathrm{p}}^{j}} & \mathbf{R}_{\mathrm{n}}^{j}
\end{array}\right]\left\{\begin{array}{l}
\boldsymbol{\alpha}_{\mathrm{p}}^{j} \\
\boldsymbol{\alpha}_{\mathrm{n}}^{j}
\end{array}\right\} .
\end{gathered}
$$

Eliminating the modal coordinates $\boldsymbol{\alpha}_{\mathrm{p}}^{j}$ and $\boldsymbol{\alpha}_{\mathrm{n}}^{j}$ from equations (33) and (34) yields:

$$
\begin{aligned}
\left\{\begin{array}{c}
\mathbf{F}_{\mathrm{L}}^{j} \\
\mathbf{F}_{\mathrm{R}}^{j}
\end{array}\right\}= & {\left[\begin{array}{cc}
\mathbf{R}_{\mathrm{p}}^{j} & -\mathbf{R}_{\mathrm{n}}^{j} e^{N_{j} \mu_{\mathrm{n}}^{j}} \\
-\mathbf{R}_{\mathrm{p}}^{j} e^{-N_{j} \mu_{\mathrm{p}}^{j}} & \mathbf{R}_{\mathrm{n}}^{j}
\end{array}\right] } \\
& {\left[\begin{array}{cc}
\boldsymbol{\Psi}_{\mathrm{p}}^{j} & \boldsymbol{\Psi}_{\mathrm{n}}^{j} e^{N_{j} \mu_{\mathrm{n}}^{j}} \\
\boldsymbol{\Psi}_{\mathrm{p}}^{j} e^{-N_{j} \mu_{\mathrm{p}}^{j}} & \boldsymbol{\Psi}_{\mathrm{n}}^{j}
\end{array}\right]\left\{\begin{array}{c}
\mathbf{u}_{\mathrm{L}}^{j} \\
\mathbf{u}_{\mathrm{R}}^{j}
\end{array}\right\} } \\
= & {\left[\begin{array}{ll}
\tilde{\mathbf{S}}_{\mathrm{LL}}^{j} & \tilde{\mathbf{S}}_{\mathrm{LR}}^{j} \\
\tilde{\mathbf{S}}_{\mathrm{RL}}^{j} & \tilde{\mathbf{S}}_{\mathrm{RR}}^{j}
\end{array}\right]\left\{\begin{array}{c}
\mathbf{u}_{\mathrm{L}}^{j} \\
\left.\mathbf{u}_{\mathrm{R}}^{j}\right\}
\end{array}\right.}
\end{aligned}
$$

with $\tilde{\mathbf{S}}^{j}$ the dynamic stiffness matrix of part $j$ after condensation of the degrees of freedom that do not belong to the left-hand or right-hand boundary of this part of the track.

When a force $\mathbf{F}$ is applied on one of the cells of part $j$, this part of the track is split into three sub-parts (figure 3): the cell on which the force is applied (part $j \mathrm{~B}$ ), the cells of part $j$ left of this cell (part $j \mathrm{~A}$ ) and the cells of part $j$ right of this cell (part $j \mathrm{C}$ ). Part $j \mathrm{~A}$ or $j \mathrm{C}$ is not present when the force is on the first or last cell of part $j$, respectively. Dynamic stiffness matrices $\tilde{\mathbf{S}}^{j \mathrm{~A}}$ and $\tilde{\mathbf{S}}^{j \mathrm{C}}$ can be computed with equation (35), using $\mathrm{N}_{j \mathrm{~A}}$ cells in part $j \mathrm{~A}$ and $\mathrm{N}_{j \mathrm{C}}$ cells in part $j \mathrm{C}$ instead of $\mathrm{N}_{j}$. If the force is applied on a cell of part 1, this part is split into the semi-infinite part $1 \mathrm{~A}$ and the finite parts $1 \mathrm{~B}$ and 1C. Similarly, if a force is applied on a cell of part $M$, this part is split into the finite parts MA and MB and the semi-infinite part MC.

Assembling the stiffness matrices of the different parts of the track, imposing continuity of displacements and equilibrium of forces at the interfaces, results in the following system of equations: 


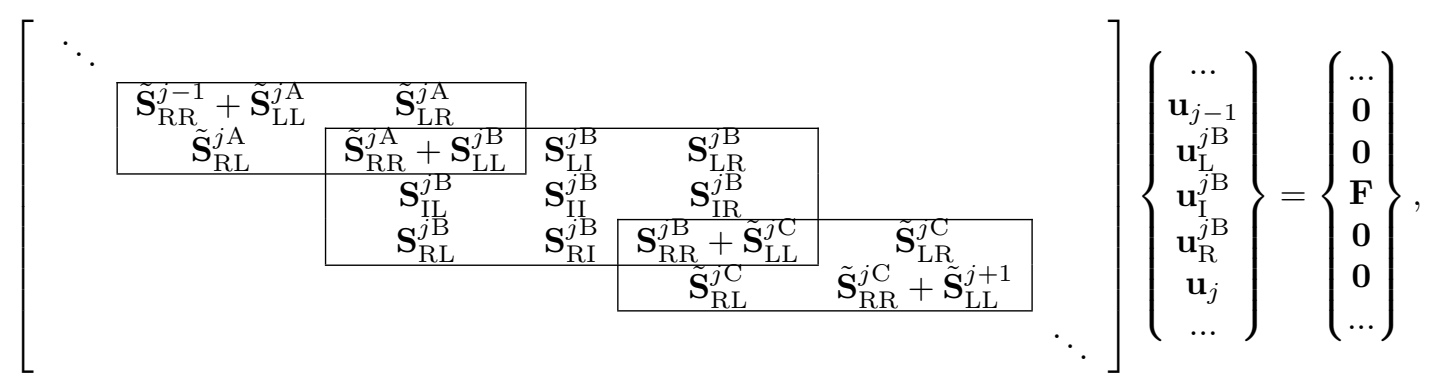

with $\mathbf{u}_{\mathrm{L}}^{j \mathrm{~B}}, \mathbf{u}_{\mathrm{I}}^{j \mathrm{~B}}$ and $\mathbf{u}_{\mathrm{R}}^{j \mathrm{~B}}$ the displacements at the left, interior and right of part $j \mathrm{~B}$ and $\mathbf{u}_{1}$ to $\mathbf{u}_{\mathrm{M}-1}$ the displacements of interfaces 1 to $M-1$. The forces and displacements inside each part of the track can be calculated from the modal coordinates in that part, using equations (29) and (33). In the particular case when the force is applied on the first cell of part $j$, part $j$ A does not exist and $\mathbf{u}_{j-1}=\mathbf{u}_{\mathrm{L}}^{j \mathrm{~B}}$. The matrix $\tilde{\mathbf{S}}_{\mathrm{RR}}^{j-1}$ is then added directly to $\mathbf{S}_{\mathrm{LL}}^{j \mathrm{~B}}$ in equation (36). Similarly, if the force is applied on the last cell of part $j$, part $j \mathrm{C}$ does not exist, $\mathbf{u}_{j}=\mathbf{u}_{\mathrm{R}}^{j \mathrm{~B}}$ and the matrix $\tilde{\mathbf{S}}_{\mathrm{LL}}^{j+1}$ is added directly to $\mathbf{S}_{\mathrm{RR}}^{j \mathrm{~B}}$.

The force $\mathbf{F}$ is subsequently applied on the different parts of the track. When the force is moving on part $j$, only the matrices $\tilde{\mathbf{S}}^{j \mathrm{~A}}$ and $\tilde{\mathbf{S}}^{j \mathrm{C}}$ change. This is exploited by performing a Gaussian elimination on the left-hand side of part $j$ until interface $j-1$ by means of the following recursive relation:

$$
\mathbf{S}_{\mathrm{R}}^{l}=\tilde{\mathbf{S}}_{\mathrm{RR}}^{l}-\tilde{\mathbf{S}}_{\mathrm{RL}}^{l}\left(\mathbf{S}_{\mathrm{R}}^{l-1}+\tilde{\mathbf{S}}_{\mathrm{LL}}^{l}\right)^{-1} \tilde{\mathbf{S}}_{\mathrm{LR}}^{l},
$$

with $l=2$ to $j-1$, resulting in the stiffness matrix $\mathbf{S}_{\mathrm{R}}^{j-1}$. Similarly, Gaussian elimination is performed on the right-hand side of part $j$ until interface $j$ by means of the following recursive relation:

$$
\mathbf{S}_{\mathrm{L}}^{l}=\tilde{\mathbf{S}}_{\mathrm{LL}}^{l}-\tilde{\mathbf{S}}_{\mathrm{LR}}^{l}\left(\mathbf{S}_{\mathrm{L}}^{l+1}+\tilde{\mathbf{S}}_{\mathrm{RR}}^{l}\right)^{-1} \tilde{\mathbf{S}}_{\mathrm{RL}}^{l},
$$

with $l=M-1$ to $j+1$, resulting in the stiffness matrix $\mathbf{S}_{\mathrm{L}}^{j+1}$. A similar Gaussian elimination is then performed for each cell of part $j$ until the left and right boundary of part $j \mathrm{~B}$ using the matrices $\tilde{\mathbf{S}}^{j \mathrm{~A}}$ and $\tilde{\mathbf{S}}^{j \mathrm{C}}$.

Next, the force enters part $j+1$. At the left side of this part, the stiffness matrix $\mathbf{S}_{\mathrm{R}}^{j}$ is obtained by Gaussian elimination until interface $j$ using equation (37) with $l=j$. At the right side, the stiffness matrix $\mathbf{S}_{\mathrm{L}}^{j+2}$ is obtained by a back substitution until interface $j+1$ :

$$
\mathbf{S}_{\mathrm{L}}^{l}=\tilde{\mathbf{S}}_{\mathrm{RL}}^{l-1}\left(\tilde{\mathbf{S}}_{\mathrm{LL}}^{l-1}-\mathbf{S}_{\mathrm{L}}^{l-1}\right)^{-1} \tilde{\mathbf{S}}_{\mathrm{LR}}^{l-1}-\tilde{\mathbf{S}}_{\mathrm{RR}}^{l-1},
$$

with $l=j+2$. Alternatively, the results $\mathbf{S}_{\mathrm{L}}^{l}$ with $l=$ $j+2$ to $M$ from recursion (38) can be stored to use afterwards when the force moves from left to right.

Since it is not possible to use the wave analysis approach with boundary elements, part of the soil underneath the track is modelled with quadratic finite elements. At the edge of the soil domain, a perfectly matched layer $(\mathrm{PML})^{23}$ is placed to absorb waves travelling in the $x$ - and $z$-direction (figure 5). The element size of the soil is chosen such that the distance between two nodes is always smaller than one eighth of the wavelength in the soil for each frequency, both in the regular soil domain and in the PML. The depth of the regular soil domain is at least equal to two wavelengths in the soil, while the width is chosen such that the distance between the ballast and the PML is at least one wavelength.

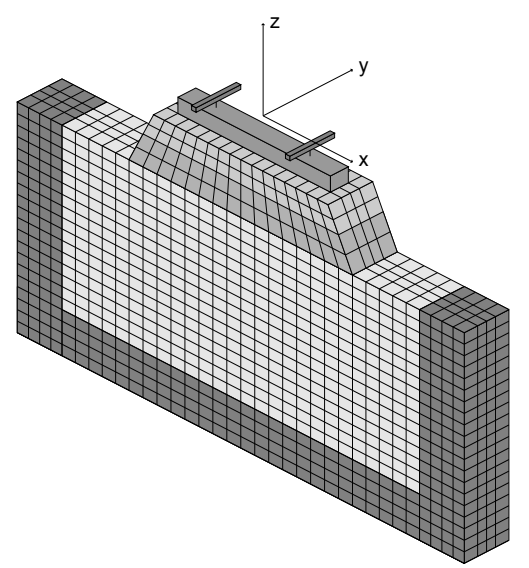

Figure 5. Model of one cell with perfectly matched layer.

To absorb the waves travelling towards the edges of the soil domain, stretched coordinates are considered for the nodes inside the PML. If $s$ denotes the coordinate in a direction normal to the interface between the physical domain and the PML located at $\mathrm{s}_{0}$, the stretched coordinate $\tilde{s}$ is defined as ${ }^{29,30}$ :

$$
\tilde{s}=s_{0}+\int_{s_{0}}^{s} \lambda_{\mathrm{s}}(s) \mathrm{d} s .
$$

The stretching functions $\lambda_{\mathrm{S}}(s)$ are complex to cause an artificially high attenuation of waves. These functions are defined as ${ }^{29,31-33}$ :

$$
\lambda_{\mathrm{s}}(s)=1+f_{\mathrm{s}}^{\mathrm{e}}(s)-i \frac{f_{\mathrm{s}}^{\mathrm{p}}(s)}{a_{0}} .
$$

The dimensionless frequency $a_{0}$ is defined as $a_{0}=$ $\omega L_{\mathrm{PML}} / C_{\mathrm{s}}$, with $L_{\mathrm{PML}}$ the thickness of the PML layer and $C_{\mathrm{s}}$ the shear wave velocity of the soil. The attenuation functions $f_{\mathrm{s}}^{\mathrm{e}}(s)$ and $f_{\mathrm{s}}^{\mathrm{p}}(s)$ attenuate evanescent and propagating waves in the $s$-direction, respectively. In order to obtain a non-reflective interface, they are equal to zero on the interface between the physical domain and the PML. Basu and Chopra ${ }^{32}$ suggest linear attenuation functions $f_{\mathrm{s}}^{\mathrm{e}}(s)$ and $f_{\mathrm{S}}^{\mathrm{p}}(s)$ :

$$
\begin{aligned}
f_{\mathrm{s}}^{\mathrm{e}}(s) & =f_{\mathrm{s} 0}^{\mathrm{e}} \frac{s}{L_{\mathrm{PML}}} \\
f_{\mathrm{s}}^{\mathrm{p}}(s) & =f_{\mathrm{s} 0}^{\mathrm{p}} \frac{s}{L_{\mathrm{PML}}} .
\end{aligned}
$$


The parameters $f_{\mathrm{s} 0}^{\mathrm{e}}$ and $f_{\mathrm{s} 0}^{\mathrm{p}}$ can be chosen to adjust the amount of attenuation inside the PML.

\section{Case study: transition zone}

The track model based on a wave analysis technique for multi-coupled periodic structures is used to model a transition zone between a ballasted track and a slab track. Slab tracks are often used on bridges and in tunnels, due to their structural and operational advantages over ballasted tracks ${ }^{34,35}$. This case study is derived from Galvín et al. ${ }^{6}$. Since a slab track has higher stiffness than a ballasted track, the transition between both track systems must be carefully designed to obtain a gradual change in track stiffness. The longitudinal geometry of a transition zone with gradually increasing stiffness is shown in figure 6 . Four parts can be distinguished: the ballasted track, the transition ballasted track, the transition slab track and the slab track. Therefore, four different types of cells must be modelled, each consisting of a finite element model of the superstructure and part of the soil, with a PML at the boundary of the soil domain as shown in figure 5. All parts of the track contain two UIC60 rails with a mass per unit length of $60.2 \mathrm{~kg} / \mathrm{m}$ and a bending stiffness of $6.45 \mathrm{Nm}^{2}$. The rail pad stiffness and damping are equal to $150 \mathrm{MN} / \mathrm{m}$ and $13.5 \mathrm{kNs} / \mathrm{m}$, respectively. The sleepers have a mass of $300 \mathrm{~kg}$ and are $2.50 \mathrm{~m}$ long and $0.235 \mathrm{~m}$ wide with spacing $L=0.60 \mathrm{~m}$. The soil is modelled as a homogeneous halfspace with shear wave velocity $C_{\mathrm{s}}$ and longitudinal wave velocity $C_{\mathrm{p}}$ equal to $150 \mathrm{~m} / \mathrm{s}$ and $300 \mathrm{~m} / \mathrm{s}$, respectively, material damping ratios $\beta_{\mathrm{s}}$ and $\beta_{\mathrm{p}}$ in shear and dilatational deformation equal to 0.04 and a density $\rho$ of $1800 \mathrm{~kg} / \mathrm{m}^{3}$.

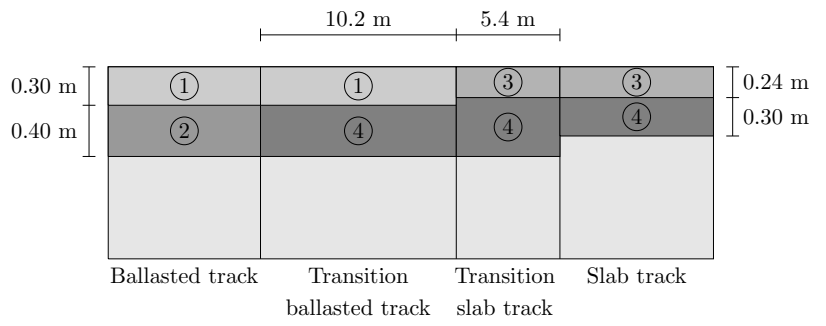

Figure 6. Longitudinal geometry of the transition zone.

The ballasted track consists of a ballast and a subballast layer. In the transition ballasted track, the subballast is replaced by layer of hydraulic subbase. The transition slab track consists of a $0.24 \mathrm{~m}$ thick concrete slab on top of a $0.46 \mathrm{~m}$ thick layer of hydraulic subbase. In the slab track, the subbase layer thickness is reduced to $0.30 \mathrm{~m}$. The properties of the materials used in the superstructure are summarized in table 1 . The dimensions of the ballasted track, transition ballasted track, transition slab track and slab track are shown in figure 7 .

The change in static track stiffness in the transition from ballasted track to slab track is shown in figure 8, for the case of a gradually increasing stiffness and for a sudden stiffness increase between ballast and slab track, i.e. without the transition ballast track and transition
Table 1. Characteristics of the materials used in the ballasted track and slab track.

\begin{tabular}{llcccc}
\hline & & $\begin{array}{c}C_{\mathrm{s}} \\
{[\mathrm{m} / \mathrm{s}]}\end{array}$ & $\begin{array}{c}C_{\mathrm{p}} \\
{[\mathrm{m} / \mathrm{s}]}\end{array}$ & $\begin{array}{c}\beta_{\mathrm{s}}=\beta_{\mathrm{p}} \\
{[-]}\end{array}$ & $\begin{array}{c}\rho \\
{\left[\mathrm{kg} / \mathrm{m}^{3}\right]}\end{array}$ \\
\hline 1 & Ballast & 278.9 & 455.4 & 0.03 & 1500 \\
2 & Sub-ballast & 197.2 & 332.0 & 0.03 & 1500 \\
3 & Slab & 2380.5 & 3887.3 & 0.03 & 2500 \\
4 & Hydraulic & 1291.0 & 2108.2 & 0.03 & 2500 \\
& subbase & & & & \\
\hline
\end{tabular}

(a)

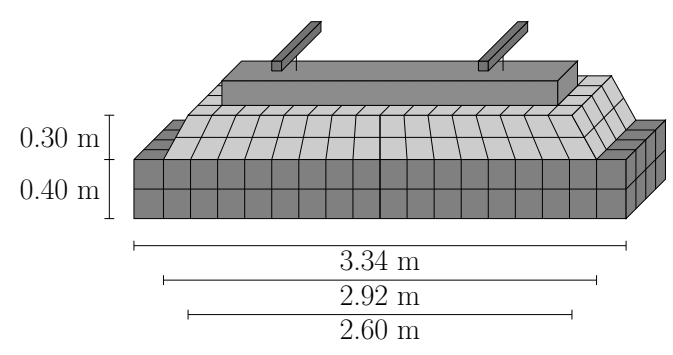

(b)

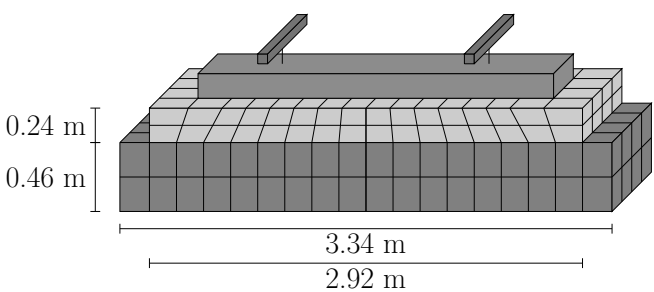

(c)

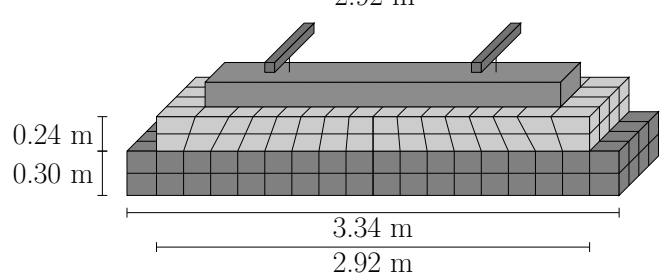

Figure 7. Model of (a) ballasted track and transition ballasted track, (b) transition slab track and (c) slab track.

slab track. This figure shows that the transition zone with gradually increasing stiffness leads to a stiffness increase in two steps, while for the transition zone with sudden stiffness increase this happens in one step. Remark that in this transition zone, the transition slab track is stiffer than the track slab itself, due to the thicker layer of hydraulic subbase. The nearby presence of the ballasted track, however, ensures that the static stiffness in the transition slab track does not exceed the stiffness of the slab track. Making the transition slab track longer would therefore not make the stiffness increase in the transition zone more gradual, but would lead to a section with higher static stiffness compared to the slab track, which is undesirable. The oscillations in the track stiffness in figure 8 are due to the periodic supports of the rails.

The superstructure and soil are modelled with 20node quadratic 3D volume elements. The mesh of the physical soil domain and PML are frequency-dependent in order to accurately model the wave propagation in the soil and to effectively absorb the waves in the PML for all frequencies. The size of the PML decreases with the frequency: 4 elements are used below $20 \mathrm{~Hz}, 3$ elements between $20 \mathrm{~Hz}$ and $50 \mathrm{~Hz}$ and 2 elements above $50 \mathrm{~Hz}$. 


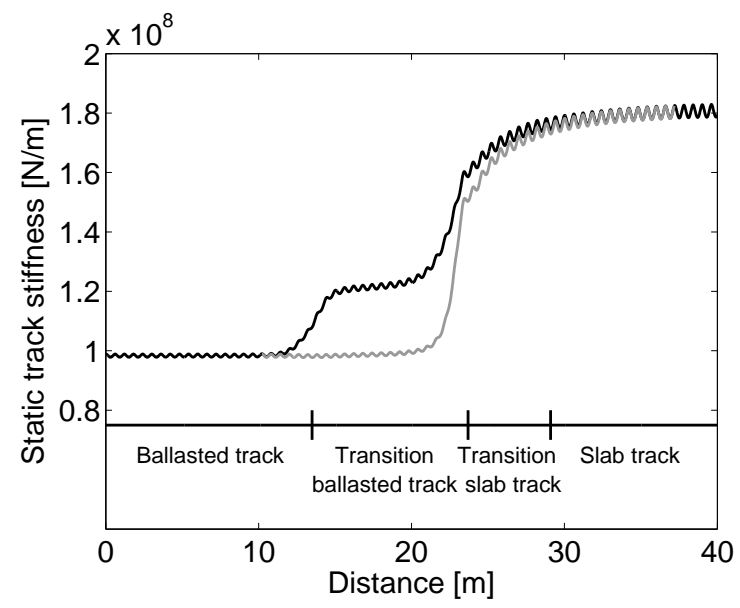

Figure 8. Static track stiffness in the transition zone from ballasted track to slab track with gradually (black line) and suddenly (grey line) increasing stiffness.

The element size in the soil $l_{\mathrm{e}}$ is equal to $C_{\mathrm{s}} /(4 f)$. In this verification, the parameter $f_{\mathrm{s} 0}^{\mathrm{e}}$ is chosen as follows in order to ensure accurate absorption of evanescent waves:

$$
f_{\mathrm{s} 0}^{\mathrm{e}}=\frac{C_{\mathrm{s}}}{l_{\mathrm{e}} f}-1
$$

The constant value $f_{\mathrm{s} 0}^{\mathrm{p}}=20$ is used, as suggested by Basu and Chopra ${ }^{32}$.

The use of the proposed track model based on a wave analysis technique for multi-coupled periodic structures is first verified. This is done for the case of a perfectly periodic track corresponding to the ballast track of the case study. The results obtained with this track model are compared with the results obtained with a periodic FE-BE model. In this case, all cells in the track model with PML are identical to allow for a comparison between two periodic track models.

Figure 9 shows the modulus and phase of the vertical rail receptance, computed above a sleeper with the two track models. The agreement is very good for the modulus. For the phase, there is a small difference for frequencies above $60 \mathrm{~Hz}$.

Figure 10 shows the modulus of the track-free field mobility at $6 \mathrm{~m}$ and $24 \mathrm{~m}$ from the track center line. The results agree very well. For frequencies above $60 \mathrm{~Hz}$, the track model with PML has a slightly higher trackfree field mobility, but the difference is never larger than $1 \mathrm{~dB}$. The difference is due to very small wave reflection on the boundaries of the soil domain and can be reduced by considering a larger PML layer.

This verification shows that the track model based on the wave analysis technique gives very similar results as a periodic FE-BE model. The computation time of these models is compared on a PC with $8 \mathrm{~GB}$ RAM and a $2.10 \mathrm{GHz}$ processor. The periodic FEBE model on average needs $1100 \mathrm{~s}$ per frequency, while the track model based on the wave analysis technique only needs $105 \mathrm{~s}$. Therefore, the latter is much more efficient despite its larger finite element grid. This is mainly due to two reasons. First, the wave analysis technique avoids the computation of the (a)

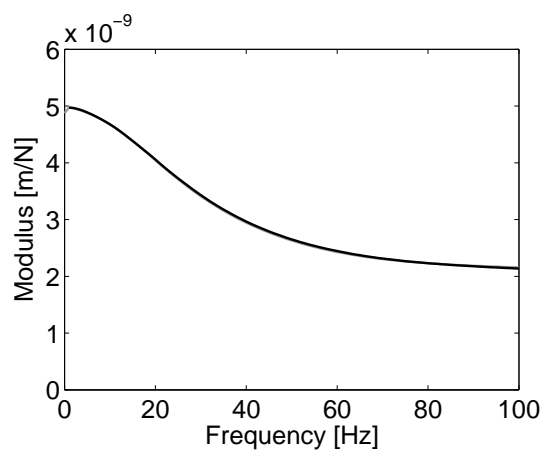

(b)

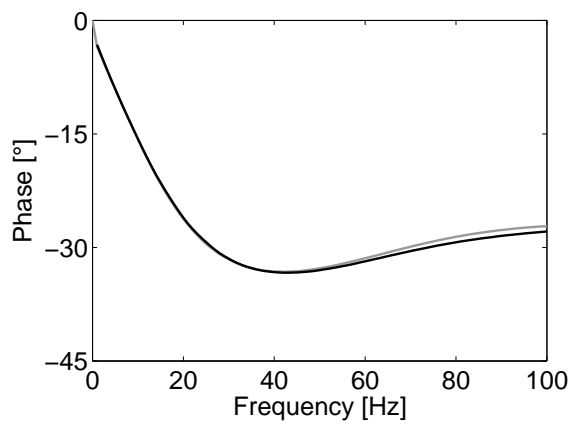

Figure 9. (a) Modulus and (b) phase of the vertical rail receptance computed with the track model with PML based on the wave analysis technique (black line) and the periodic FE-BE track model (grey line)

(a)

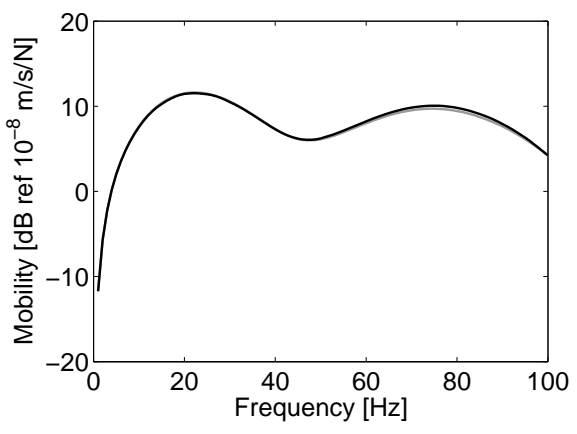

(b)

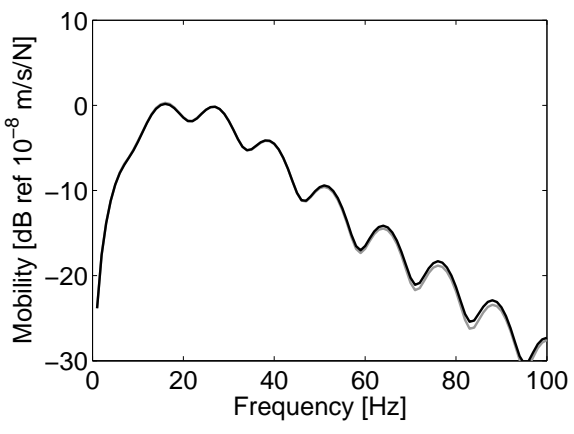

Figure 10. Modulus of the track-free field mobility at (a) $6 \mathrm{~m}$ and (b) $24 \mathrm{~m}$ from the track center line computed with the track model with PML based on the wave analysis technique (black line) and the periodic FE-BE track model (grey line).

computationally very expensive soil stiffness matrices. Also, the equations in the periodic FE-BE model must be solved for every wavenumber and frequency, while in the wave analysis technique all computations are solved only once for each frequency. The highest additional 


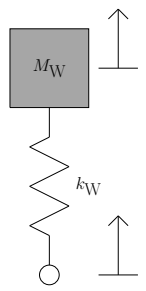

Figure 11. Model of a Thalys high speed train wheel set.

cost of the wave analysis technique is the solution of the eigenvalue problem of equation (25). Based on the comparison between the computation times of the periodic FE-BE model and FE model with PML, it can be concluded that the wave analysis technique also significantly reduces the computation time compared to a 3D FE-BE model.

The train-track interaction force and free field vibrations for a train passing on the transition zone are computed subsequently using the algorithm described in subsection 2.1. In order to make the interpretation of the results more easy, the train is represented by a single wheelset of a Thalys high speed train. The wheelset model consists of a mass $M_{\mathrm{w}}$ of $2027 \mathrm{~kg}$ and a massless wheel-rail contact point (figure 11). The mass $M_{\mathrm{w}}$ and the massless contact point are connected by a spring $k_{\mathrm{w}}$ with stiffness $2.80 \times 10^{9} \mathrm{~N} / \mathrm{m}$. The total mass carried by the axle is equal to $20 \times 10^{3} \mathrm{~kg}$. Three vehicle speeds are considered: 100, 200 and $300 \mathrm{~km} / \mathrm{h}$, resulting in a sleeper passage frequency of $46.3 \mathrm{~Hz}, 92.6 \mathrm{~Hz}$ and $138.9 \mathrm{~Hz}$, respectively.

To evaluate the effectiveness of the transition zone, the passage of the vehicle is simulated for a transition zone with gradually increasing stiffness, corresponding to figure 6, and for a track with a sudden stiffness increase, the vehicle thus moving directly from a ballasted track to a slab track. Figures 12 to 14 show the time history and frequency spectrum of the train-track interaction force for the three considered train speeds. In the time domain, the results are synchronized in such a way that the change from ballasted track to slab track in the track with sudden stiffness change occurs at the same moment as the change from transition ballasted track to transition slab track in the track with gradual stiffness change. The periodicity of the interaction force, corresponding to the sleeper passage frequencies, can clearly be observed. The highest interaction forces due to the periodic rail support are observed at 100 $\mathrm{km} / \mathrm{h}$. This can be explained by figure 15, showing the receptance of the vehicle and the track. Around $50 \mathrm{~Hz}$, vehicle and track have approximately the same receptance, which corresponds to the so-called P2-resonance frequency at which higher train-track interaction forces are expected. At $100 \mathrm{~km} / \mathrm{h}$, the sleeper passage frequency is close to this resonance frequency, giving rise to higher dynamic excitation. It is also observed that the periodic excitation is higher for the slab track than for the ballasted track, due to the stiffer behavior of the former.

The effect of the transition zone is limited at 100 $\mathrm{km} / \mathrm{h}$ and $200 \mathrm{~km} / \mathrm{h}$. At $300 \mathrm{~km} / \mathrm{h}$, however, higher dynamic excitation is clearly observed passing from ballasted to slab track if the train experiences a sudden track stiffness increase. In the frequency spectrum, this gives rise to higher forces in a broad frequency band, with a peak around the P2-resonance frequency of the train-track system. Figure 16 shows the spectrograms of the interaction force with a time window of $0.02 \mathrm{~s}$. This figure clearly shows the change from ballasted track to slab track in the case with sudden stiffness increase in the transition zone, and the changes from ballasted track to transition ballasted track and from the latter to transition slab track for the track with gradual stiffness increase in the transition zone. The same color scale is used in both figures. Force levels are clearly reduced when a transition zone with gradually increasing stiffness is installed.

Apart from parametric excitation, wheel and rail unevenness also contribute to the dynamic excitation of the train and track. To model this unevenness excitation, a stochastic track unevenness profile $u_{\mathrm{w} / \mathrm{r}}(y)$ is generated as the superposition of harmonic functions with random phase angle $\theta_{i}$ uniformly distributed in the interval $[0,2 \pi]^{25}$ :

$$
u_{\mathrm{w} / \mathrm{r}}(y)=\sum_{i=1}^{n} \alpha_{i} \cos \left(k_{y i} y-\theta_{i}\right),
$$

where the parameters $\alpha_{i}$ are determined by imposing that the mean square of the artificial profile $u_{\mathrm{w} / \mathrm{r}}(y)$ is equal to the area under the power spectral density (PSD) curve $\tilde{G}_{\mathrm{w} / \mathrm{r}}\left(k_{y i}\right)$ in each interval $\Delta k_{y i}$ with center wavenumber $k_{y i}$ :

$$
\alpha_{i}=\sqrt{2 G_{\mathrm{w} / \mathrm{r}}\left(k_{y i}\right) \Delta k_{y}} .
$$

The following expression is used for the one-sided PSD function ${ }^{36}$ :

$$
\tilde{G}_{\mathrm{w} / \mathrm{r}}\left(k_{y}\right)=\tilde{G}_{\mathrm{w} / \mathrm{r}}\left(k_{y 0}\right)\left(\frac{k_{y}}{k_{y 0}}\right)^{-\mathrm{w}}
$$

where $k_{y 0}=1 \mathrm{rad} / \mathrm{m}$ and $w=3.5$. According to the ISO 8608 standard, the lower and upper limits of $\tilde{G}_{\mathrm{w} / \mathrm{r}}\left(k_{y 0}\right)$ are $1 \times 10^{-9} \mathrm{~m}^{3}$ and $5 \times 10^{-7} \mathrm{~m}^{3}$, respectively. For a high speed train and track, unevenness is usually very low. Therefore, the lower limit value of $1 \times 10^{-9} \mathrm{~m}^{3}$ is used.

A gradual change in track stiffness is required most at $300 \mathrm{~km} / \mathrm{h}$. Figure 17 shows the time history and frequency spectrum of the train-track interaction force at this speed, taking into account wheel and rail unevenness. Particularly high dynamic forces are observed between 40 and $60 \mathrm{~Hz}$, corresponding to frequencies close to the $\mathrm{P} 2$ resonance frequency. The unevenness excitation now has an important contribution to the dynamic excitation. In the time history, the peaks due to the transition from ballast to concrete slab can be observed. Higher peaks are observed in the case with sudden stiffness increase in the transition zone, about two times higher than those due to the unevenness excitation. Therefore, the transition from ballasted to slab track has an important influence 
(a)

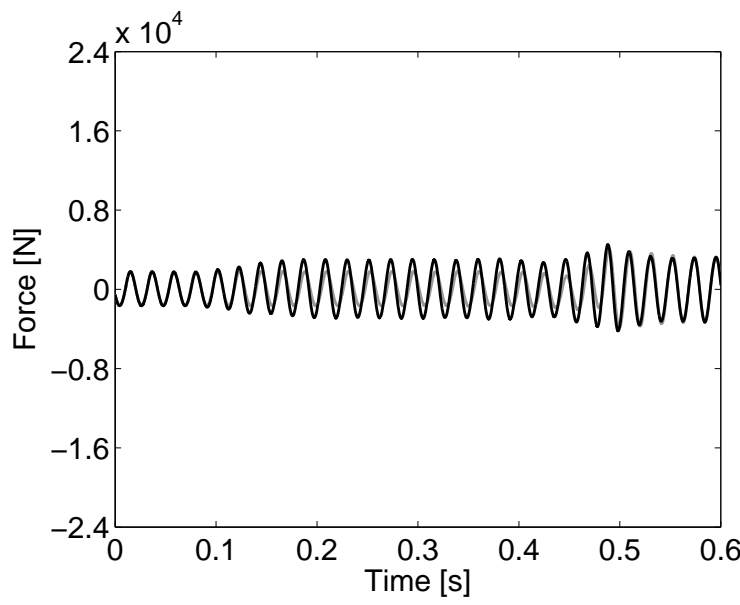

(b)

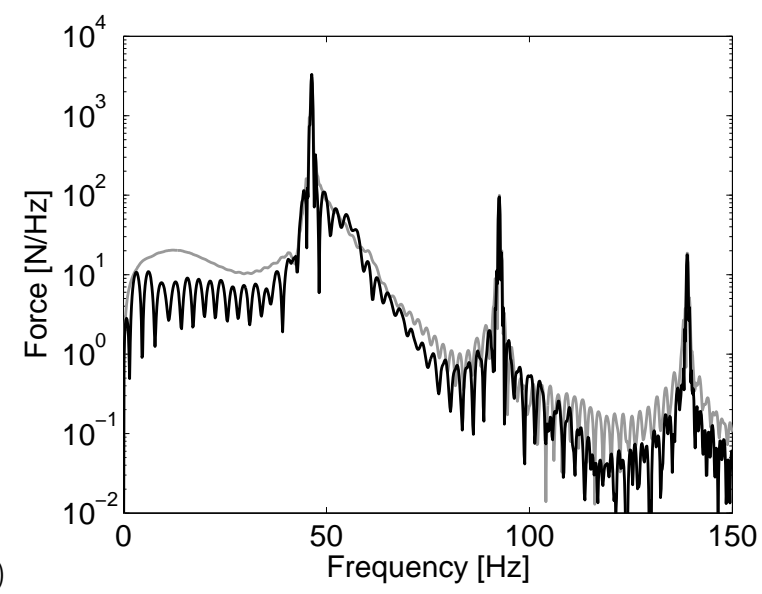

Figure 12. (a) Time history and (b) frequency spectrum of the dynamic train-track interaction force for the passage of the wheel set at $100 \mathrm{~km} / \mathrm{h}$ on the track with gradually (black line) and suddenly (grey line) increasing stiffness in the transition zone.

(a)

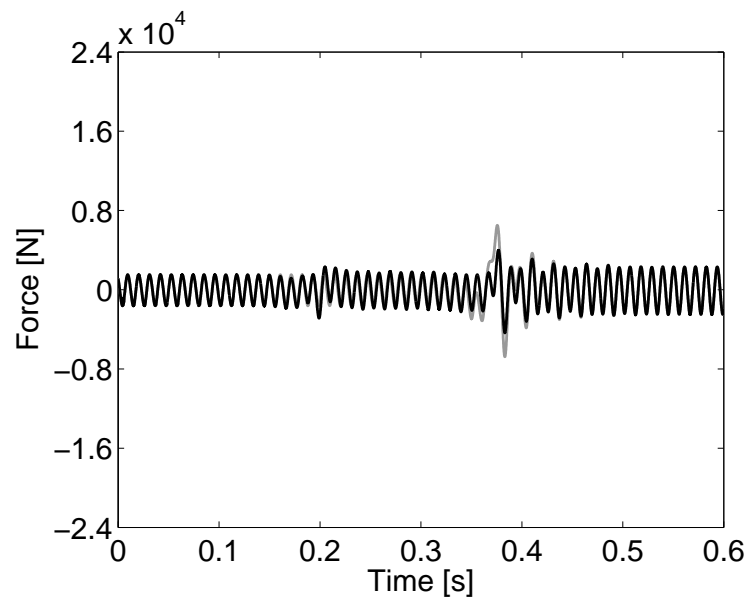

(b)

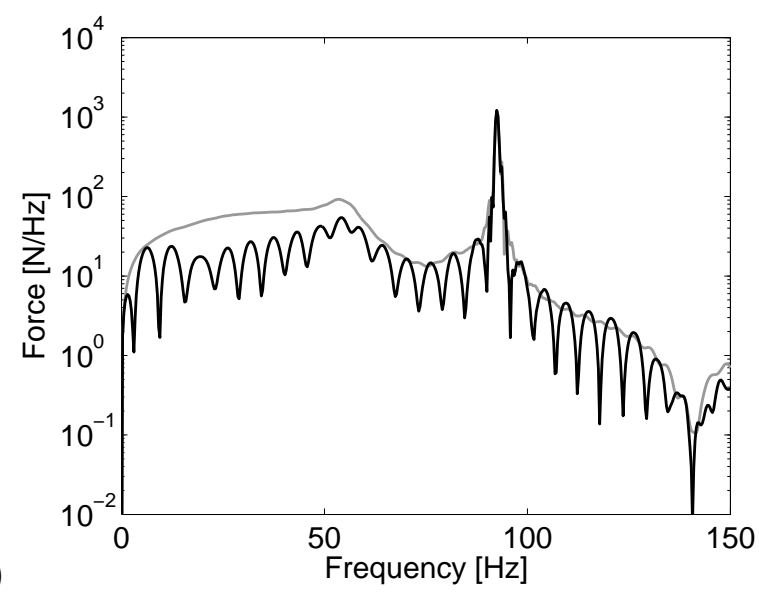

Figure 13. (a) Time history and (b) frequency spectrum of the dynamic train-track interaction force for the passage of the wheel set at $200 \mathrm{~km} / \mathrm{h}$ on the track with gradually (black line) and suddenly (grey line) increasing stiffness in the transition zone.

(a)

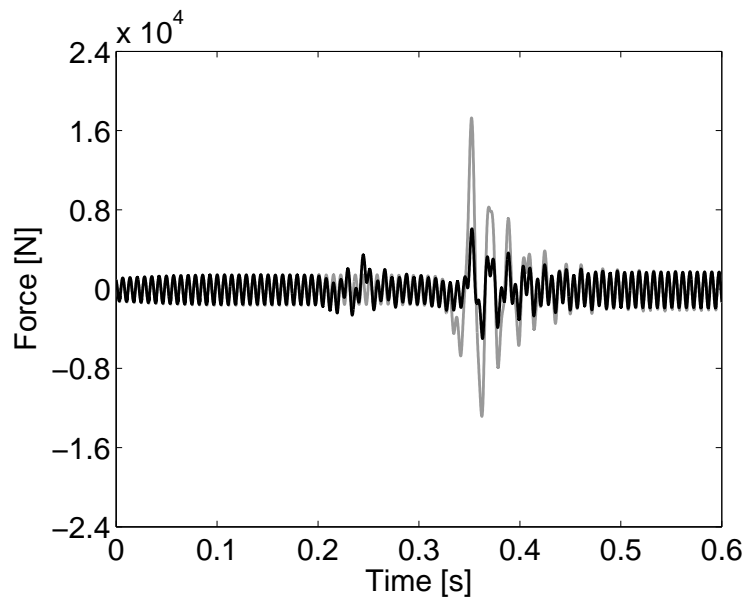

(b)

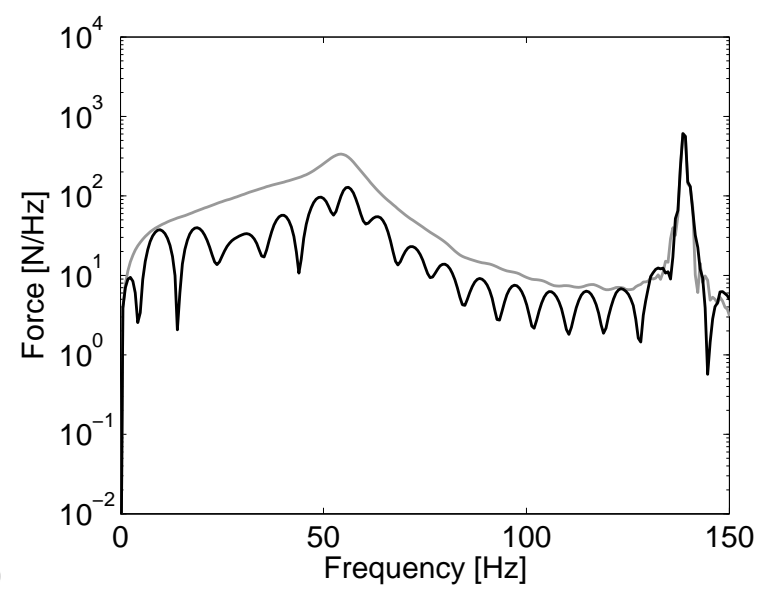

Figure 14. (a) Time history and (b) frequency spectrum of the dynamic train-track interaction force for the passage of the wheel set at $300 \mathrm{~km} / \mathrm{h}$ on the track with gradually (black line) and suddenly (grey line) increasing stiffness in the transition zone.

on the force applied on the track, which will lead to faster degradation of the track structure at the location of the transition. In the frequency spectrum of the force, almost no difference can be observed since the difference in interaction force occurs only for a very short period of time. Therefore, the transition zone will have a relatively small influence on the free field vibrations when wheel and rail unevenness are introduced. This is because in this case study, the unevenness profile was generated randomly and independently from the track structure. In reality, the stiffness change at the transition between ballasted and slab track will result in higher interaction 


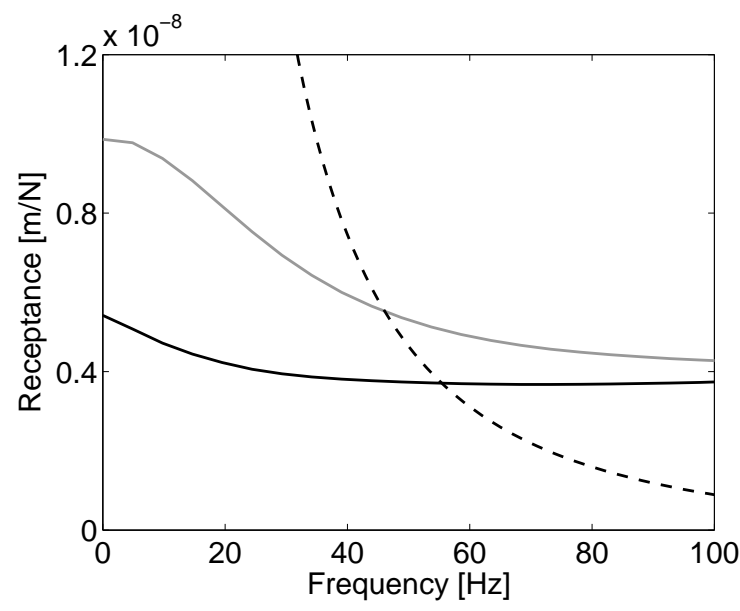

Figure 15. Receptance of the slab track (solid black line), ballasted track (solid grey line) and vehicle (dashed black line).

(a)
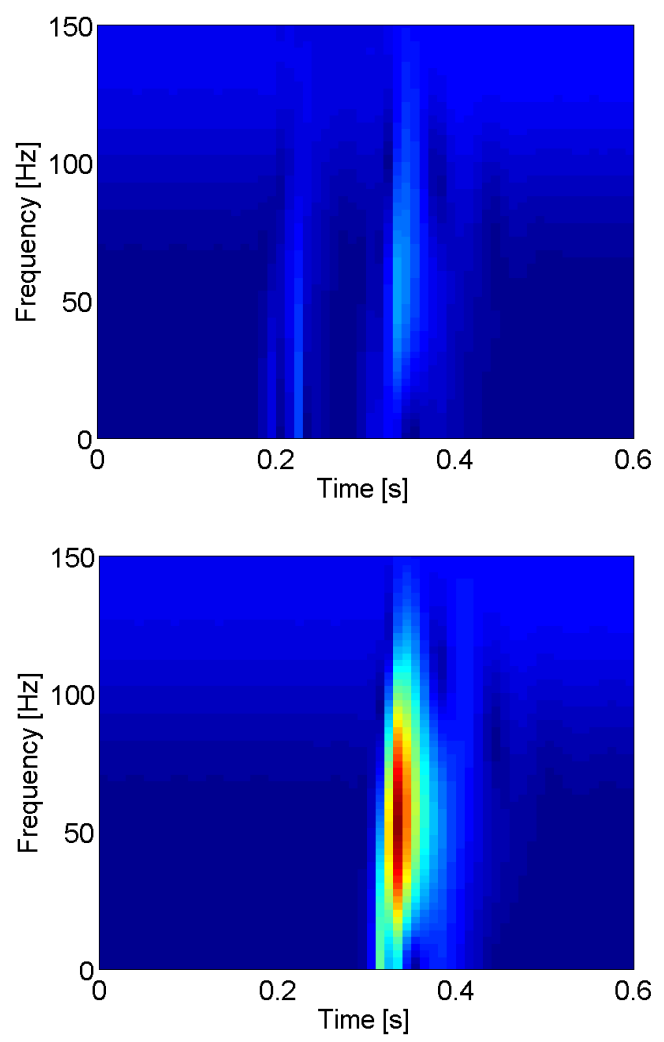

Figure 16. Spectrogram of the dynamic train-track interaction force at a train speed of $300 \mathrm{~km} / \mathrm{h}$ for a track with (a) gradually and (b) suddenly increasing stiffness in the transition zone.

forces, leading to differential settlements in this region. In that way, the change in track stiffness and the unevenness will amplify each other.

The dynamic train-track interaction forces are subsequently used to predict the free field response. The interaction forces at $300 \mathrm{~km} / \mathrm{h}$ are used, since at this speed the influence of the transition zone was most important. Wheel and rail unevenness are disregarded. Figures 18 to 20 show the time history and frequency spectrum of the free field velocity at $6 \mathrm{~m}, 12 \mathrm{~m}$ and $24 \mathrm{~m}$ of the track center line at the longitudinal position of the transition between the transition ballasted track and transition slab track. In case of a sudden stiffness increase in the transition zone, much higher free field vibrations are observed, up to $0.42 \mathrm{~mm} / \mathrm{s}$ at $6 \mathrm{~m}$ from the track center line. The sudden change in track stiffness leads to an impulse load on the track. The vibrations are transferred through the track structure and the soil to the free field, where the arrival of the Pwave and Rayleigh wave can be clearly observed as the first and second peak, respectively. The sudden stiffness increase in the transition zone leads to higher vibrations for all frequencies between 0 and $120 \mathrm{~Hz}$. The peak at the sleeper passage frequency is still present in the spectrum; this periodic vibration is also observed in the time history.

\section{Conclusion}

This paper presents a track modelling approach based on a wave analysis technique for multi-coupled periodic structures. Wave reflection on the boundaries of the soil domain is avoided by a perfectly matched layer at the boundaries of the soil domain. The advantage of this technique is that it is computationally efficient, and unlike a $2.5 \mathrm{D}$ or periodic model allows to model a track with varying characteristics in the longitudinal direction. For coupling a train model to this track model, a two-step train-track-soil coupling algorithm is presented.

The track model based on a wave analysis technique for multi-coupled periodic structures is used to model a transition zone between a ballasted track and a slab track. The proposed track model is verified by a comparison with a periodic FE-BE model. A periodic ballasted track is therefore modelled with both techniques. The vertical rail receptance and track-free field mobility are compared and the results of both models are shown to correspond very well. Next, the passage of a train on the transition zone is computed for a track with a gradually and suddenly increasing stiffness, respectively. The train-track interaction force and free field vibrations are compared. A sudden stiffness increase in the transition zone leads to higher interaction forces for all frequencies up to $150 \mathrm{~Hz}$. This effect is stronger with increasing train speed. In the free field, the sudden stiffness increase in the transition zone leads to much higher vibrations for frequencies up to $120 \mathrm{~Hz}$.

\section{Acknowledgements}

The first author is a PhD student supported by the Research Foundation Flanders (FWO). The financial support is gratefully acknowledged. 
(a)

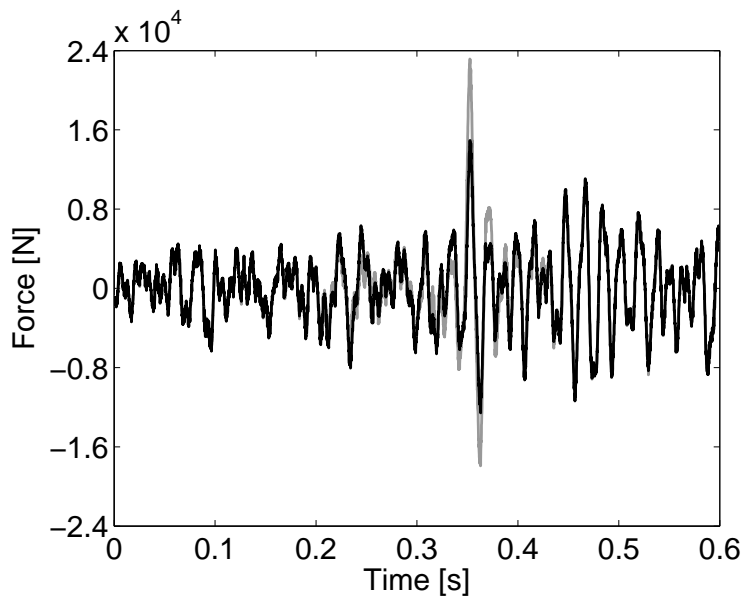

(b)

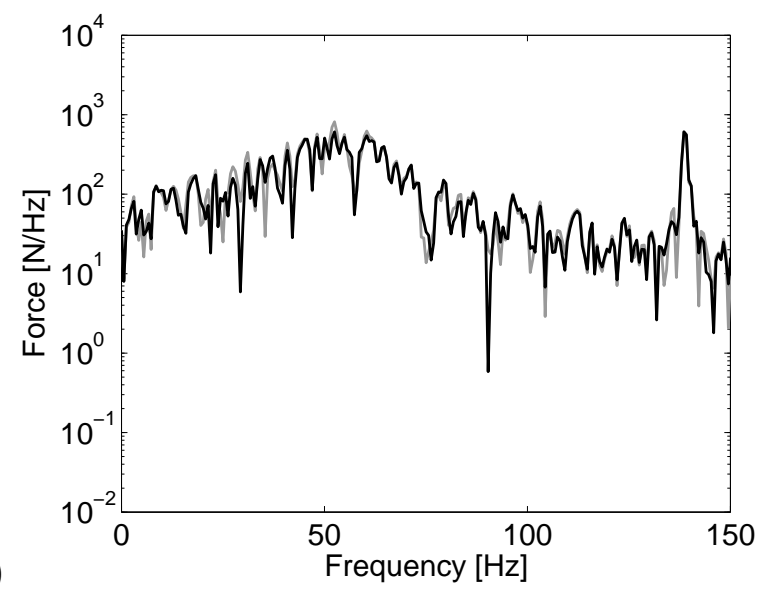

Figure 17. (a) Time history and (b) frequency spectrum of the dynamic train-track interaction force taking into account wheel and rail unevenness, for the passage of the wheel set at $300 \mathrm{~km} / \mathrm{h}$ on the track with gradually (black line) and suddenly (grey line) increasing stiffness in the transition zone.

(a)

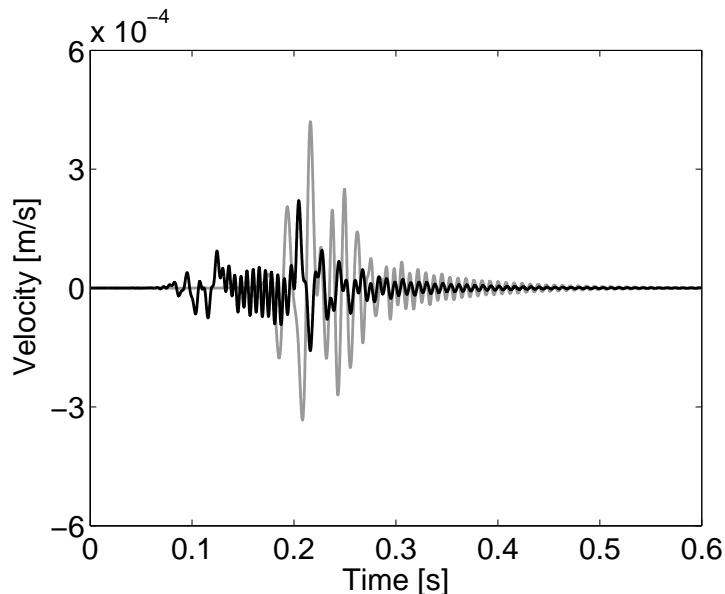

(b)

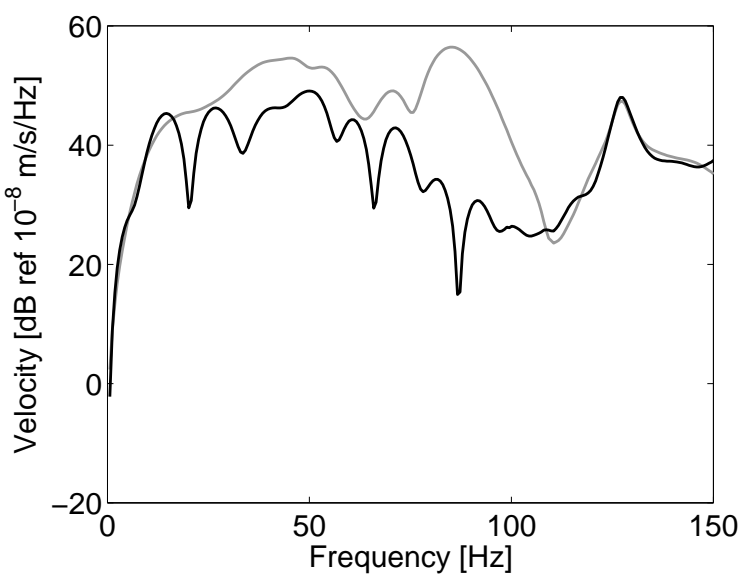

Figure 18. (a) Time history and (b) frequency spectrum of the free field velocities at $6 \mathrm{~m}$ of the track center line for the passage of the wheel set at $300 \mathrm{~km} / \mathrm{h}$ on the track with gradually (black line) and suddenly (grey line) increasing stiffness in the transition zone.

(a)

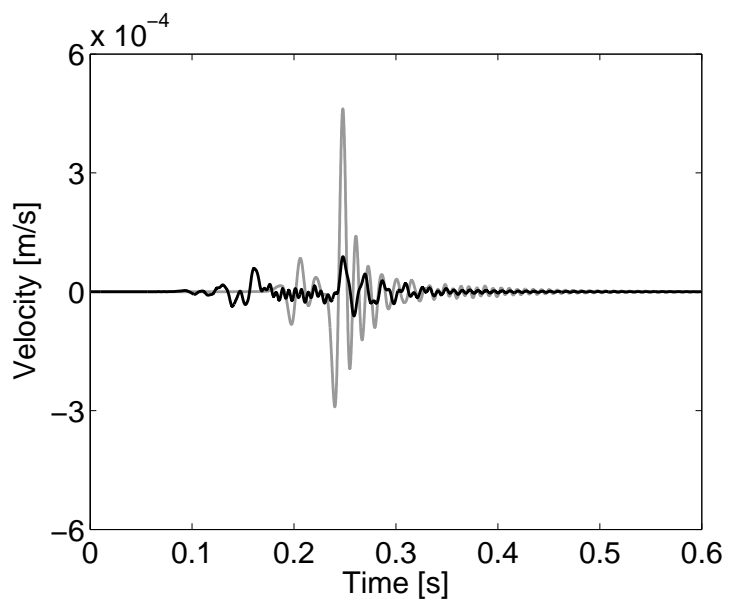

(b)

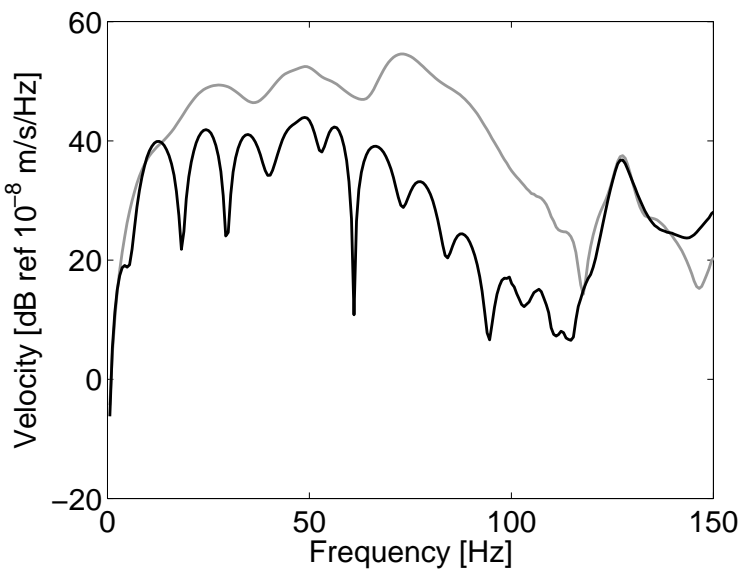

Figure 19. (a) Time history and (b) frequency spectrum of the free field velocities at $12 \mathrm{~m}$ of the track center line for the passage of the wheel set at $300 \mathrm{~km} / \mathrm{h}$ on the track with gradually (black line) and suddenly (grey line) increasing stiffness in the transition zone.

\section{References}

1. Lombaert G, Degrande G, François S et al. Groundborne vibration due to railway traffic. In Nielsen
J, Anderson D, de Vos $\mathrm{P}$ et al. (eds.) Proceedings of the 11th International Workshop on Railway Noise IWRN11. Uddevalla, Sweden, pp. 266-301. Invited state 
(a)

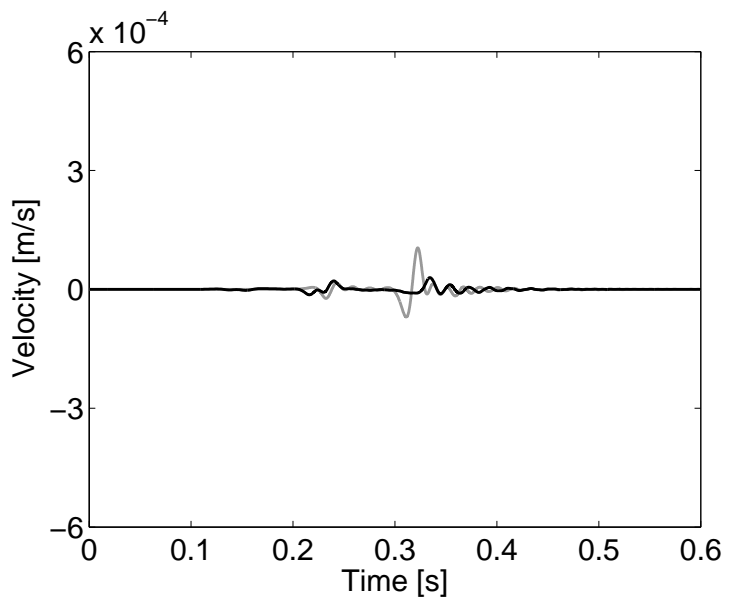

(b)

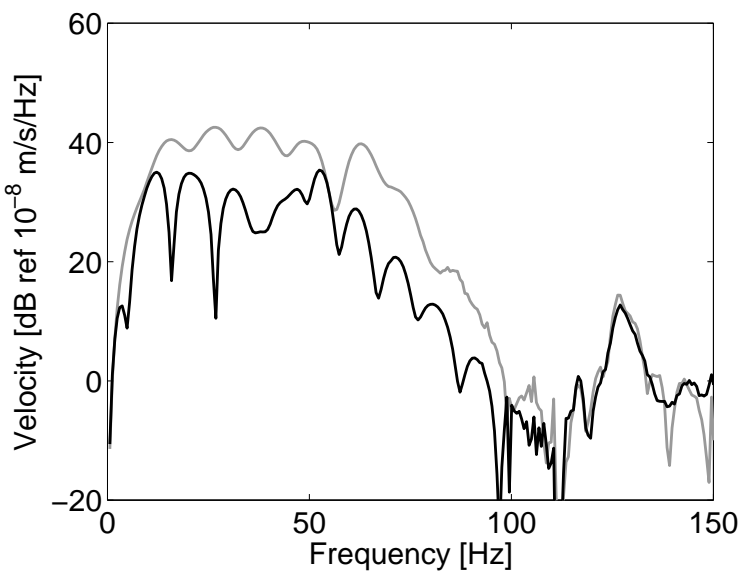

Figure 20. (a) Time history and (b) frequency spectrum of the free field velocities at $24 \mathrm{~m}$ of the track center line for the passage of the wheel set at $300 \mathrm{~km} / \mathrm{h}$ on the track with gradually (black line) and suddenly (grey line) increasing stiffness in the transition zone.

of the art paper.

2. Thompson D. Railway noise and vibration: mechanisms, modelling, and means of control. Oxford: Elsevier, 2009.

3. Heckl M, Hauck G and Wettschureck R. Structure-borne sound and vibration from rail traffic. Journal of Sound and Vibration 1996; 193(1): 175-184.

4. Knothe K and Grassie S. Modelling of railway track and vehicle/track interaction at high frequencies. Vehicle Systems Dynamics 1993; 22: 209-262.

5. Coelho B. Dynamics of railway transition zones in soft soils. PhD Thesis, TU Delft, 2011.

6. Galvín P, Romero A and Domínguez J. Fully threedimensional analysis of high-speed train-track-soilstructure dynamic interaction. Journal of Sound and Vibration 2010; 329: 5147-5163.

7. O'Brien J and Rizos D. A 3D FEM-BEM methodology for simulation of high speed train induced vibrations. Soil Dynamics and Earthquake Engineering 2005; 25: 289-301.

8. Costa P, Calçada R and Cardoso A. Ballast mats for the reduction of railway traffic vibrations. Numerical study. Soil Dynamics and Earthquake Engineering 2012; 42: 137-150.

9. François S, Schevenels M, Lombaert G et al. A 2.5D coupled FE-BE methodology for the dynamic interaction between longitudinally invariant structures and a layered halfspace. Computer Methods in Applied Mechanics and Engineering 2010; 199(23-24): 15361548.

10. Sheng X, Jones C and Thompson D. Prediction of ground vibration from trains using the wavenumber finite and boundary element methods. Journal of Sound and Vibration 2006; 293: 575-586.

11. Forrest $\mathrm{J}$ and Hunt H. A three-dimensional tunnel model for calculation of train-induced ground vibration. Journal of Sound and Vibration 2006; 294(4): 678-705.

12. Müller K, Grundmann H and Lenz S. Nonlinear interaction between a moving vehicle and a plate elastically mounted on a tunnel. Journal of Sound and Vibration 2008; 310: 558-586.
13. Clouteau D, Arnst M, Al-Hussaini T et al. Freefield vibrations due to dynamic loading on a tunnel embedded in a stratified medium. Journal of Sound and Vibration 2005; 283(1-2): 173-199.

14. Clouteau D, Elhabre M and Aubry D. Periodic BEM and FEM-BEM coupling: application to seismic behaviour of very long structures. Computational Mechanics 2000; 25: 567-577.

15. Arlaud E. Modèles dynamiques réduits de milieux périodiques par morceaux : application aux voies ferroviaires. PhD Thesis, l'École Nationale Supérieure d'Arts et Métiers, 2016.

16. Arlaud E, Costa D'Aguar S, Balmes E et al. Numerical study of railway track dynamics: Case of a transition zone. Engineering Analysis with Boundary Elements 2016; 27: 23-38.

17. Nielsen $\mathrm{J}$ and Igeland A. Vertical dynamic interaction between train and track-influence of wheel and rail imperfections. Journal of Sound and Vibration 1995; 187(5): 825-839.

18. Nielsen J, Lombaert G and François S. A hybrid model for prediction of ground-borne vibration due to discrete wheel/rail irregularities. Journal of Sound and Vibration 2015; 345: 103-120.

19. Triepaischajonsak $\mathrm{N}$ and Thompson D. A hybrid modelling approach for predicting ground vibration from trains. Journal of Sound and Vibration 2015; 335: 147173.

20. Coelho B, Hölscher P, Priest J et al. An assessment of transition zone performance. In Proceedings of the Institution of Mechanical Engineers Part F: Journal of Rail and Rapid Transit, volume 225(2). pp. 129-139.

21. Varandas J, Hölscher P and Silva M. Dynamic behaviour of railway tracks on transitions zones. Computers and Structures 2011; 89: 1468-1479.

22. Mead D. The forced vibration of one-dimensional multicoupled periodic structures: An application to finite element analysis. Journal of Sound and Vibration 2009; 319: 282-304.

23. François S, Schevenels M, Lombaert G et al. A 2.5D displacement based PML for elastodynamic wave 
propagation. International Journal for Numerical Methods in Engineering 2012; 90(7): 819-837.

24. François S, Coulier P and Degrande G. Finite elementboundary element coupling algorithms for transient elastodynamics. Engineering Analysis with Boundary Elements 2015; 55: 104-121.

25. Lombaert G, Degrande G, Kogut J et al. The experimental validation of a numerical model for the prediction of railway induced vibrations. Journal of Sound and Vibration 2006; 297(3-5): 512-535.

26. Aitken A. Studies in practical mathematics II: The evaluation of the latent roots and latent vectors of a matrix. Proceedings of the Royal Society of Edinburgh 1937; 57: 269-304.

27. Lombaert G and Degrande G. Ground-borne vibration due to static and dynamic axle loads of InterCity and high speed trains. Journal of Sound and Vibration 2009; 319(3-5): 1036-1066.

28. Lak M, François S, Degrande G et al. Development and experimental validation of a numerical model for the prediction of ground vibration generated by pavement breaking. Soil Dynamics and Earthquake Engineering 2015; 79: 199-210.

29. Chew W and Liu Q. Perfectly matched layers for elastodynamics: A new absorbing boundary condition.
Journal of Computational Acoustics 1996; 4(4): 341-359.

30. Chew W, Jin J and Michielssen E. Complex coordinate stretching as a generalized absorbing boundary condition. Microwave and Optical Technology Letters 1997; 15(6): 363-369.

31. Bérenger J. A perfectly matched layer for the absorption of electromagnetic waves. Journal of Computational Physics 1994; 41: 115-135.

32. Basu U and Chopra A. Perfectly matched layers for time-harmonic elastodynamics of unbounded domains: theory and finite-element implementation. Computer Methods in Applied Mechanics and Engineering 2003; 192(11-12): 1337-1375.

33. Basu U. Explicit finite element perfectly matched layer for transient three-dimensional elastic waves. International Journal for Numerical Methods in Engineering 2009; 77: 151-176.

34. Esveld C. Modern railway track. Second Edition. MRTProductions, Zaltbommel, 2001.

35. Steenbergen M, Metrikine A and Esveld C. Assessment of design parameters of a slab track railway system from a dynamic viewpoint. Journal of Sound and Vibration 2007; 306: 361-371.

36. Braun $\mathrm{H}$ and Hellenbroich T. Messergebnisse von Strassenunebenheiten. VDI Berichte 1991; 877: 47-80. 